\title{
The RNA-binding protein tristetraprolin schedules apoptosis of pathogen-engaged neutrophils during bacterial infection
}

\author{
Florian Ebner, ${ }^{1}$ Vitaly Sedlyarov, ${ }^{1}$ Saren Tasciyan, ${ }^{2}$ Masa Ivin, ${ }^{1}$ Franz Kratochvill, ${ }^{1}$ Nina Gratz, ${ }^{1}$ Lukas Kenner, ${ }^{3,4,5}$ \\ Andreas Villunger, ${ }^{6,7}$ Michael Sixt, $^{2}$ and Pavel Kovarik ${ }^{1}$ \\ 'Max F. Perutz Laboratories, University of Vienna, Vienna, Austria. Institute of Science and Technology Austria (IST Austria), Klosterneuburg, Austria. ${ }^{3}$ Clinical Institute of Pathology, Medical University of \\ Vienna, Vienna, Austria. ${ }^{4}$ Ludwig Boltzmann Institute for Cancer Research, Vienna, Austria. ${ }^{5}$ Unit of Pathology of Laboratory Animals (UPLA), University of Veterinary Medicine Vienna, Vienna, Austria. \\ ${ }^{6}$ Medical University of Innsbruck, Division of Developmental Immunology, Innsbruck, Austria. ${ }^{7}$ Tyrolean Cancer Research Institute, Innsbruck, Austria.
}

\begin{abstract}
Protective responses against pathogens require a rapid mobilization of resting neutrophils and the timely removal of activated ones. Neutrophils are exceptionally short-lived leukocytes, yet it remains unclear whether the lifespan of pathogen-engaged neutrophils is regulated differently from that in the circulating steady-state pool. Here, we have found that under homeostatic conditions, the mRNA-destabilizing protein tristetraprolin (TTP) regulates apoptosis and the numbers of activated infiltrating murine neutrophils but not neutrophil cellularity. Activated TTP-deficient neutrophils exhibited decreased apoptosis and enhanced accumulation at the infection site. In the context of myeloid-specific deletion of Ttp, the potentiation of neutrophil deployment protected mice against lethal soft tissue infection with Streptococcus pyogenes and prevented bacterial dissemination. Neutrophil transcriptome analysis revealed that decreased apoptosis of TTP-deficient neutrophils was specifically associated with elevated expression of myeloid cell leukemia 1 (Mc/1) but not other antiapoptotic B cell leukemia/ lymphoma 2 (Bc/2) family members. Higher Mcl1 expression resulted from stabilization of Mcl1 mRNA in the absence of TTP. The low apoptosis rate of infiltrating TTP-deficient neutrophils was comparable to that of transgenic Mcl1-overexpressing neutrophils. Our study demonstrates that posttranscriptional gene regulation by TTP schedules the termination of the antimicrobial engagement of neutrophils. The balancing role of TTP comes at the cost of an increased risk of bacterial infections.
\end{abstract}

\section{Introduction}

Neutrophils are life-saving antimicrobial immune cells and the most abundant blood leukocytes. They are rapidly mobilized and the first host defense cells to infiltrate the site of infection. Neutrophils directly protect the host by a number of mechanisms that include degranulation, pathogen phagocytosis, generation of ROS, production of antimicrobial peptides, or formation of neutrophil extracellular traps (NETs) (1). Indirect defense mechanisms include the secretion of inflammation-amplifying cytokines and chemokines. Recent findings revealed that neutrophils also act as a communication interface that helps coordinate tissue regeneration and activation of adaptive responses $(1,2)$. The high damaging potential of neutrophils is kept under control mainly by a limited lifespan (2-4). Although it is well established that apoptosis is the main control mechanism of neutrophil numbers, the temporal regulation of this central process of neutrophil homeostasis is not understood $(2,4)$.

mRNA stability is known to play an important role in the control of immune responses (5), yet its role in neutrophil homeostasis and regulation of the immune defense against infections is unex-

Conflict of interest: The authors have declared that no conflict of interest exists Submitted: December 19, 2014; Accepted: March 16, 2017.

Reference information: / Clin Invest. 2017;127(6):2051-2065.

https://doi.org/10.1172/JCl80631. plored. The immune response commences with a marked stabilization of inflammation-associated mRNAs, including cytokine and chemokine mRNAs, whereas a timely and efficient degradation of such transcripts highlights the resolution phase of inflammation (5, 6). mRNA stability is controlled by cis-acting regulatory elements located mostly in the 3 '-UTRs and trans-acting RNA-binding proteins and miRs $(5,7)$. Tristetraprolin (TTP, encoded by Ttp, also known as $Z f p 36$ ) is an RNA-binding and -destabilizing protein that targets preferentially AU-rich elements (AREs) in 3'-UTRs for degradation by recruiting the CCR4-NOT deadenylase and the decapping complex (8). A multifaceted and still incompletely understood regulation of TTP by transcriptional, posttranscriptional, and posttranslational mechanisms orchestrates TTP activity, such that an efficient degradation occurs in the resolution phase of inflammation, and a premature degradation of target mRNAs is avoided (8-13). Ttp-knockout mice are born healthy but suffer from granulocytic hyperplasia and develop a progressive and eventually lethal pleiotropic inflammation (14). The inflammatory symptoms are reduced upon blockage of TNF or IL-23 $(14,15)$, but it remains possible that these cytokines act together or downstream of other defects resulting from TTP deficiency. Mice with $L y s M$-Cre-driven Ttp deletion in macrophages and neutrophils are healthy despite an increased susceptibility to endotoxin-mediated septic shock $(12,16)$. The role of TTP during bacterial infection and its specific function in neutrophils remain unknown. 
In this study, we show that TTP robustly regulates the neutrophil transcriptome and that this regulation is qualitatively and quantitatively different from the TTP-dependent control of the macrophage transcriptome. Neutrophil TTP attenuates the expression of genes involved in critical antimicrobial functions of neutrophils such as infiltration and the lifespan. Upon induction of bacterial peritonitis, the elicited TTP-deficient neutrophils were increased in numbers and exhibited reduced myeloid cell leukemia 1-controlled (Mcl1-controlled) apoptosis. mRNA of Mcl1, which is an essential antiapoptotic gene in neutrophils $(17,18)$, was bound and destabilized by TTP. TTP deficiency did not affect neutrophils under nonchallenged conditions, demonstrating that TTP restricts neutrophils that are engaged in host-pathogen interactions. In a model of severe invasive infection with Streptococcus pyogenes, a leading gram-positive human pathogen $(19,20)$, the enhanced engagement of TTP-deficient neutrophils prevented systemic dissemination of the pathogen and improved the survival of infected animals. These results establish that neutrophil deployment caused by a microbial insult is temporally limited by TTP-mediated apoptosis. Inhibition of this feedback mechanism results in an improved resistance against lethal bacterial infection of the soft tissue.

\section{Results}

TTP ablation in myeloid cells protects the host against invasive bacterial infection by preventing bacterial dissemination. Tissuespecific deletion of Ttp using Tt $p^{f l f l} L y s M$-Cre animals (referred to herein as $\mathrm{TTP}^{\Delta \mathrm{M}}$ mice) demonstrated an essential function for myeloid TTP expression in the protection against LPS-induced toxic shock $(12,16)$, but the role of TTP in immune responses to live pathogens has not been elucidated. To this end, we used a mouse model of invasive soft tissue infection with $S$. pyogenes. In this model, animals experience spreading necrosis, followed by bacteremia and mortality closely resembling severe necrotizing fasciitis in humans $(21,22)$. We investigated TTP function in this infection model by using $\mathrm{TTP}^{\Delta \mathrm{M}}$ mice, which lack TTP in neutrophils, monocytes, and macrophages (12), all of which are essential for defense against $S$. pyogenes infection (23-26). Infection of $T t p^{f / f l}$ control animals (referred to herein as $\mathrm{TTP}^{\mathrm{fl} / \mathrm{fl}}$ mice) led to $90 \%$ lethality (humane endpoint) 5 days post infection (p.i.), whereas only $20 \%$ of the $\mathrm{TTP}^{\Delta \mathrm{M}}$ littermates succumbed to the infection (Figure 1A). Animals that survived 5 days showed signs of recovery. The lesion that formed at the site of injection 48 hours p.i. had an approximately $40 \%$ larger surface area in $\mathrm{TTP}^{\Delta \mathrm{M}}$ mice than in $\mathrm{TTP}^{\mathrm{fl} / \mathrm{fl}}$ littermates $\left(0.81 \pm 0.21 \mathrm{~cm}^{2}\right.$ and 0.58 $\pm 0.14 \mathrm{~cm}^{2}$, respectively) (Figure 1B). Histopathological analysis of H\&E-stained sections of the lesion showed an elevated inflammatory reaction in the $\mathrm{TTP}^{\Delta \mathrm{M}}$ animals (Figure $1, \mathrm{C}$ and D). Notably, necrosis, infiltration of leukocytes, and the overall grading were significantly increased in $\mathrm{TTP}^{\Delta \mathrm{M}}$ animals compared with controls 48 hours p.i. Gr-1 staining for $\mathrm{Ly}_{6 \mathrm{G}} \mathrm{G}^{+}$and/or Ly6C $\mathrm{C}^{+}$ myeloid cells in the lesion sections confirmed the presence of high numbers of myeloid cells in the infiltrate of $\mathrm{TTP}^{\triangle \mathrm{M}}$ mice (Figure 1D). The infiltrate colocalized with S. pyogenes bacteria, which were well contained in $\mathrm{TTP}^{\Delta \mathrm{M}}$ lesions, but spreading in the $\mathrm{TTP}^{\mathrm{fl} / \mathrm{fl}}$ specimens (Figure 1D). Mainly focal localization of bacteria at the site of inoculation, together with increased animal survival, suggested that $\mathrm{TTP}^{\Delta \mathrm{M}}$ mice were able to restrict systemic dissemination of the invading $S$. pyogenes. This was confirmed by CFU assays, which revealed a dramatically lower bacterial burden in the blood and liver of $\mathrm{TTP}^{\Delta \mathrm{M}}$ mice when compared with that detected in $\mathrm{TTP}^{\mathrm{fl} / \mathrm{fl}}$ littermates, but a similar bacterial burden at the site of primary infection (i.e., skin) in both genotypes (Figure $1 \mathrm{E})$. Serum levels of urea, creatinine, aspartate transaminase (AST), alanine transaminase (ALT), glutamate dehydrogenase (GLDH), and creatine kinase (CK) were low and comparable between $\mathrm{TTP}^{\Delta \mathrm{M}}$ and $\mathrm{TTP}^{\mathrm{fl} / \mathrm{fl}}$ mice 48 hours p.i. (Supplemental Figure 1A; supplemental material available online with this article; https://doi.org/10.1172/JCI80631DS1), implying no gross differences and/or impairment in organ function. These data are in agreement with lethal sepsis following uncontrolled bacterial dissemination rather than direct organ injury as the cause of death in this infection model $(22,27,28)$.

In sum, $\mathrm{TTP}^{\Delta \mathrm{M}}$ mice launch a stronger inflammatory response against invading bacteria than do their control littermates. Despite more severe tissue damage at the site of infection, the enhanced response is beneficial to the $\mathrm{TTP}^{\Delta \mathrm{M}}$ host, as it prevents dissemination of $S$. pyogenes.

The reservoir of myeloid cells is not regulated by TTP during infection. The enhanced leukocyte deployment and immune response during infection of $\mathrm{TTP}^{\Delta \mathrm{M}}$ animals with S.pyogenes might be caused by a larger reservoir of myeloid cells. $\mathrm{TTP}^{\Delta \mathrm{M}}$ and $\mathrm{TTP}^{\mathrm{fl} / \mathrm{ll}}$ mice were reported to possess comparable numbers of circulating myeloid cells under steady-state conditions $(12,16)$. Similarly, analysis of resident peritoneal macrophages $\left(\mathrm{F} 4 / 80^{\mathrm{hi}}\right)$, which are of the same origin as skin-resident macrophages (29), revealed comparable numbers in $\mathrm{TTP}^{\Delta \mathrm{M}}$ and $\mathrm{TTP}^{\mathrm{fl} / \mathrm{fl}}$ mice under steady-state conditions (Supplemental Figure 1, B and C). These data indicated that TTP regulates neither circulating nor tissue-resident myeloid cells under homeostatic conditions.

To investigate myeloid cells under conditions of infection, we focused on key infiltrating cells, i.e., neutrophils (CD11b+Ly6 $\mathrm{G}^{+}$ Ly6 $\mathrm{C}^{+}$Siglec-F-FceRI $)$and inflammatory monocytes (CD11b ${ }^{+} \mathrm{Ly}^{-} \mathrm{G}^{-}$ Ly $6 \mathrm{C}^{\text {hi }}$ Siglec-F-FceRI'). The numbers of neutrophils and inflammatory monocytes in the blood and bone marrow were comparable in $\mathrm{TTP}^{\Delta \mathrm{M}}$ and $\mathrm{TTP}^{\mathrm{fl} / \mathrm{fl}}$ mice both prior to and 48 hours after infection (Figure 2, A and B). Consistent with the infection-elicited emigration of bone marrow neutrophils into the circulation (23), the neutrophil pool dropped in the bone marrow but increased in the blood 48 hours p.i. (Figure 2A). Infection augmented the number of inflammatory monocytes both in the bone marrow and the blood (Figure 2A), which is in line with the differentiation of Ly6 $\mathrm{C}^{\text {hi }}$ inflammatory monocytes and their release into the circulation (30). Importantly, these changes were similar in $\mathrm{TTP}^{\Delta \mathrm{M}}$ and $\mathrm{TTP}^{\mathrm{fl} / \mathrm{fl}}$ mice. Moreover, our analysis of marginated neutrophils, i.e., circulating neutrophils retarded in the vasculature of specific organs (31), in the lung and spleen revealed no differences between $\mathrm{TTP}^{\Delta \mathrm{M}}$ and $\mathrm{TTP}^{\mathrm{f} / \mathrm{fl}}$ mice (Supplemental Figure 2A). In sum, TTP did not regulate circulating neutrophils or inflammatory monocytes before or during infection.

We then asked whether TTP regulated the production of immature neutrophils, since an enhanced bone marrow granulopoiesis in $\mathrm{TTP}^{\Delta \mathrm{M}}$ mice might explain the observed increase in neutrophil deployment in infected animals (Figure 1D) if both granulopoiesis and migration of neutrophils were enhanced in 
A
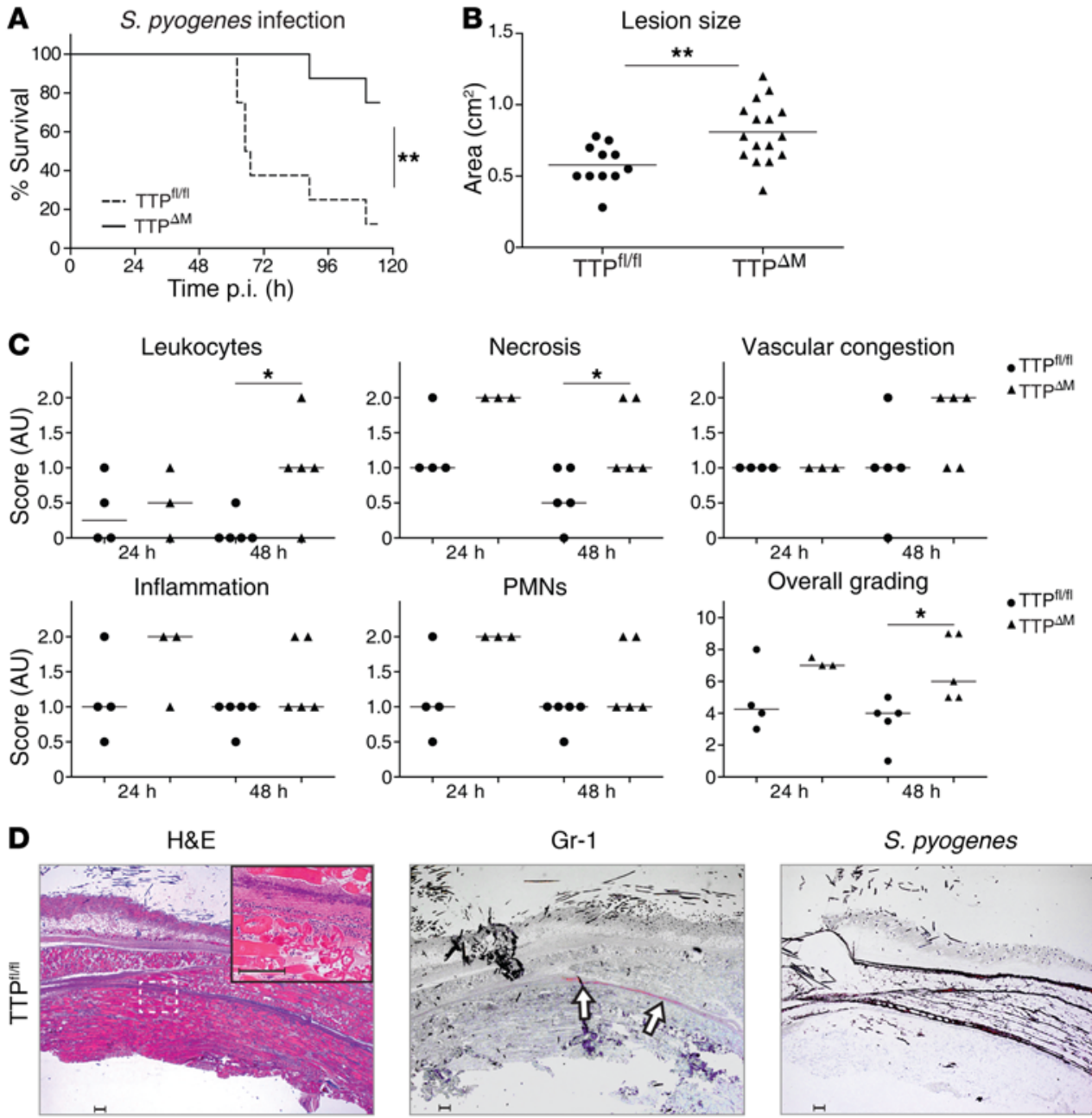

D

$H \& E$
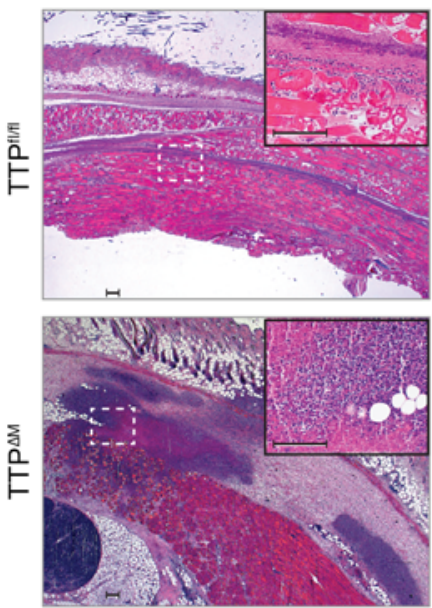

Gr-1
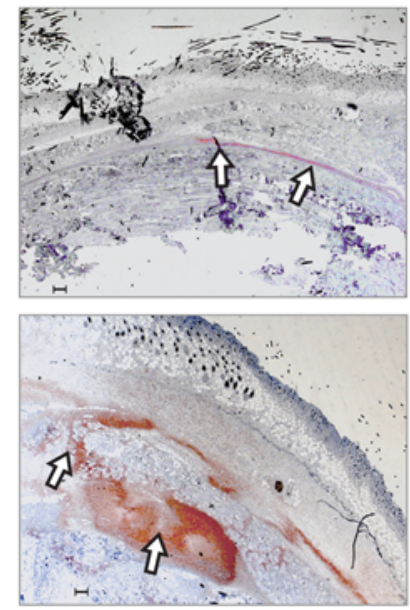
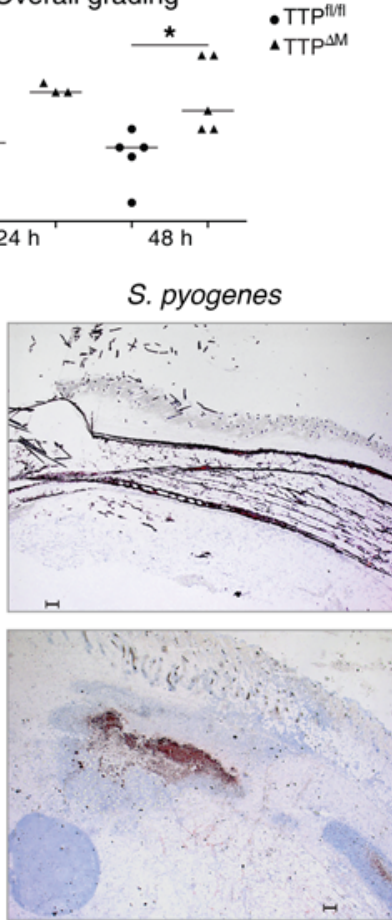

Figure 1. Myeloid-specific deletion of $T t p$ increases immune response and resistance against invasive infection with S. pyogenes. (A) Survival of $\mathrm{TTP}^{\mathrm{fl} / \mathrm{fl}}$ and TTP $\mathrm{TP}^{\Delta \mathrm{M}}$ mice ( $n=8$ /genotype) during s.c. infection with $3 \times 10^{8} \mathrm{CFU} S$. pyogenes. Animals were monitored for 5 days. Kaplan-Meier survival curves are shown. ${ }^{* *} P<0.01$, by Mantel-Cox test. (B) The area of inflammation was determined 48 hours p.i. Two independent experiments were pooled $(n=11$ $T T P^{f / / f l}$ and $n=16 T T P^{\Delta M}$ mice in total). Error bars represent the mean. ${ }^{*} P<$ 0.01 , by unpaired Student's $t$ test. (C) $\mathrm{H} \& \mathrm{E}$-stained tissue sections of flanks from TTP $P^{\mathrm{f} / \mathrm{fl}}$ and TTP $\mathrm{P}^{\Delta \mathrm{M}}$ mice 24 and 48 hours p.i. were graded for leukocyte infiltration, necrotic tissue abundance, inflammation, PMN infiltration, and vascular congestion. Scores were combined for overall grading. Error bars indicate the median. ${ }^{*} P<0.05$, by Mann-Whitney $U$ test. (D) Representative H\&E- (left panel), Gr-1- (middle panel), and S. pyogenes-stained (right panel) tissue sections of flanks from TTP ${ }^{\text {fl/fl }}$ (top) and TTP ${ }^{\Delta M}$ (bottom) animals 48 hours p.i. Arrows indicate Gr-1-stained cells. Note an increased infiltrate and containment of bacteria in tissues from TTP ${ }^{\triangle M}$ mice. Original magnification, $\times 2.5$ and $\times 20$ (insets in H\&E-stained images). Scale bars: 200 $\mu \mathrm{m}$. (E) Bacterial loads in the blood, liver, and lesions from TTP//fl and TTP ${ }^{\triangle M}$ mice infected with $3 \times 10^{8}$ CFU S. pyogenes. Samples were taken 48 hours p.i., and the bacterial load was determined. Dot plots represent a pool of 2 independent experiments ( $n=11 \mathrm{TTP}^{\mathrm{fl} / \mathrm{fl}}$ and $n=14 \mathrm{TTP}^{\Delta \mathrm{M}}$ mice for organs; $n=13 \mathrm{TTP}^{\mathrm{fl} / / \mathrm{fl}}$ and $n=11 \mathrm{TTP}^{\Delta \mathrm{M}}$ mice for lesions). Error bars indicate the median. ${ }^{*} P<0.05$ and ${ }^{* *} P<0.01$, by Mann-Whitney $U$ test.
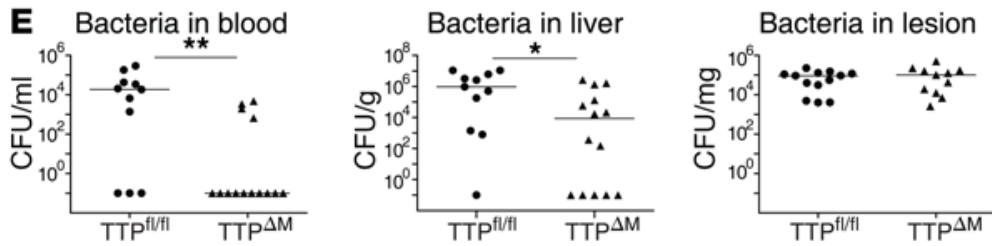

the absence of TTP. The numbers of common myeloid progenitors (CMPs) ( $\mathrm{Lin}^{-} \mathrm{Sca}-1^{-} \mathrm{c}-\mathrm{Kit}^{+} \mathrm{CD} 16 / 32^{\mathrm{lo}} \mathrm{CD} 34^{+}$) and granulocytemonocyte progenitors (GMPs) ( $\mathrm{Lin}^{-} \mathrm{Sca}-1^{-} \mathrm{c}-\mathrm{Kit}^{+} \mathrm{CD} 16 / 32^{\mathrm{hi}} \mathrm{CD} 34^{+}$) under steady-state conditions were comparable in $\mathrm{TTP}^{\Delta \mathrm{M}}$ and $\mathrm{TTP}^{\mathrm{f} / \mathrm{f}}$ mice (Figure 2C and Supplemental Figure 2B). We found that infection did not change CMP numbers, regardless of the genotype (Figure 2C and Supplemental Figure 2B). GMPs were increased to a similar extent in both genotypes upon infection (Figure 2C). The bone marrow and blood populations of mature $\left(\mathrm{Ly} 6 \mathrm{G}^{+} \mathrm{c}-\mathrm{Kit}{ }^{-}\right)$and immature $\left(\mathrm{Ly}_{6} \mathrm{G}^{+} \mathrm{c}-\mathrm{Kit}^{+}\right)$neutrophils were not regulated by TTP under steady-state conditions (Figure 2, D and E, and Supplemental Figure 2C). We also observed that infection caused mature neutrophils to drop in the bone marrow and increase in the blood, consistent with infection-facilitated neutrophil egress, but these changes were similar in $\mathrm{TTP}^{\Delta \mathrm{M}}$ and $\mathrm{TTP}^{\mathrm{f} / \mathrm{fl}}$ mice (Figure 2, D and E, and Supplemental Figure 2C). Immature neutrophils followed the same pattern upon infection, i.e., a decrease in the bone marrow and an increase in the blood, but the populations were consistently slightly higher in $\mathrm{TTP}^{\mathrm{fl} / \mathrm{fl}}$ mice than in $\mathrm{TTP}^{\Delta \mathrm{M}}$ mice $\left(\mathrm{Ly} 6 \mathrm{G}^{+} \mathrm{c}-\mathrm{Kit}^{+}\right.$bone marrow cells: mean $5.0 \%$ 
A
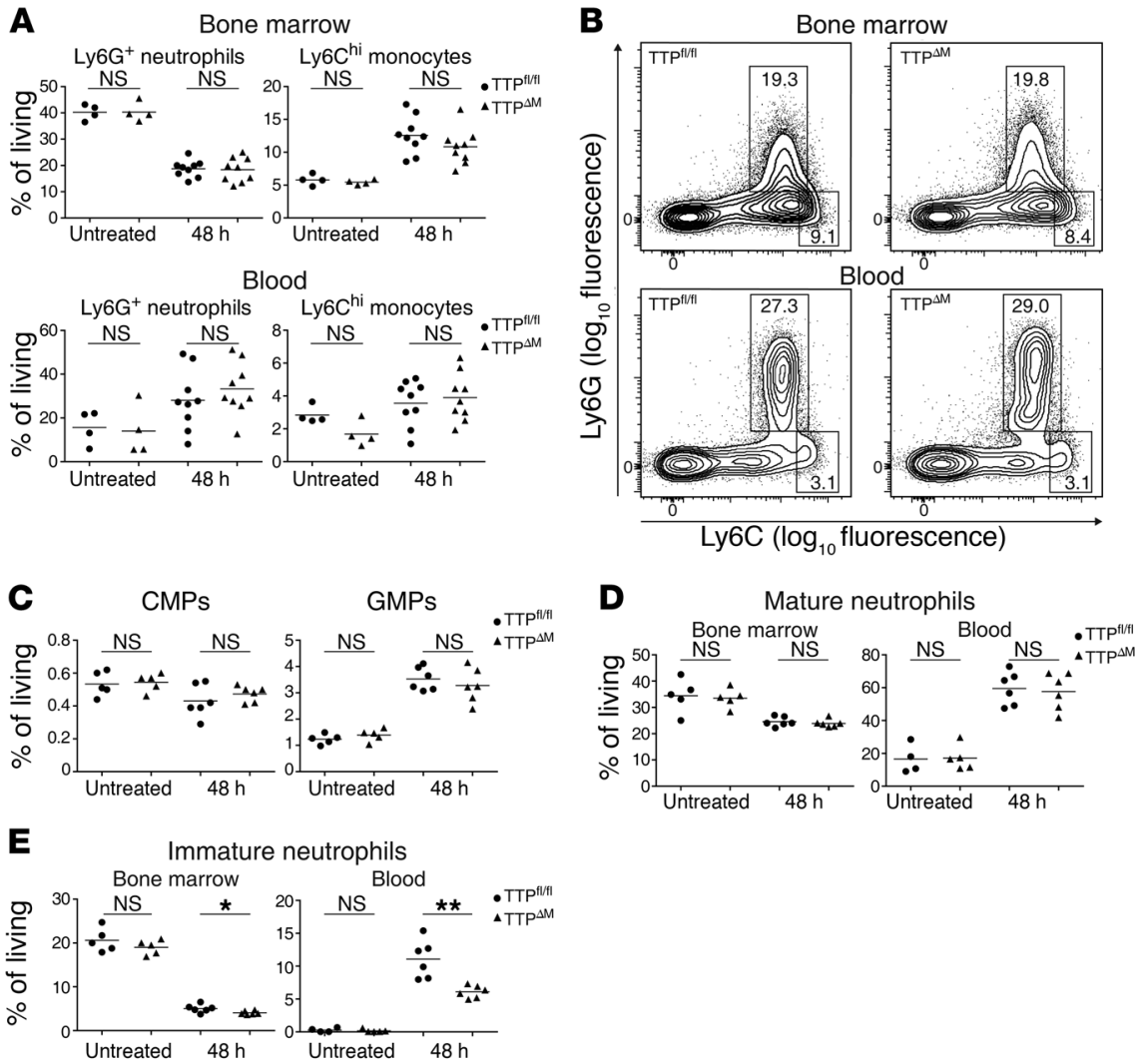

Figure 2. Bone marrow and circulating neutrophils and monocytes are not regulated by TTP during bacterial infection. ( $A$ and $\mathbf{B}$ ) Neutrophil and inflammatory monocyte populations in the bone marrow and blood of TTP ${ }^{\mathrm{fl} / \mathrm{fl}}$ and TTP $\mathrm{TP}^{\Delta \mathrm{M}}$ animals. Bone marrow and blood of untreated or infected animals were analyzed by flow cytometry 48 hours p.i. Cells were subgated for CD11b ${ }^{+}$ Siglec-F-FceRI- cell populations, and neutrophils and inflammatory monocytes were detected as Ly6C ${ }^{+} \mathrm{Ly}^{-} \mathrm{C}^{+}$or Ly6C- $\mathrm{Ly}^{-} \mathrm{C}^{\text {hi }}$ cells, respectively. Dot plots of neutrophil and inflammatory monocytes in bone marrow (A, upper panel) and blood (A, lower panel) of untreated ( $n=4$ /genotype) and infected animals ( $n=9 /$ genotype). Representative flow plots of bone marrow (B, upper panel) and blood (B, lower panel) 48 hours p.i. Numbers indicate the percentages in the outlined area.

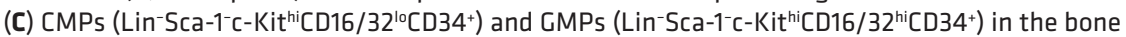
marrow of untreated and infected (48 hours p.i.) TTP ${ }^{f / / f l}$ and TTP ${ }^{\Delta M}$ mice were analyzed by flow

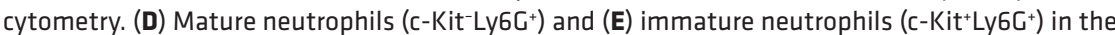
bone marrow and blood of untreated and infected (48 hours p.i.) TTP ${ }^{f / / f l}$ and TTP ${ }^{\Delta M}$ mice were analyzed by flow cytometry. $n=5$ untreated TTP ${ }^{\text {fl/fl }} ; n=5$ untreated TTP ${ }^{\Delta \mathrm{M}}$ mice; $n=6$ infected TTP fl/fl $^{\text {m }}$ mice; and $n=6$ infected TTP ${ }^{\Delta M}$ mice were used in C, D, and $\mathbf{E}$. Error bars represent the mean. ${ }^{*} P<0.05$ and ${ }^{* *} P<0.01$, by unpaired Student's $t$ test.

in $\mathrm{TTP}^{\mathrm{fl} / \mathrm{fl}} \mathrm{vs.} 4.0 \%$ in $\mathrm{TTP}^{\Delta \mathrm{M}}$ mice; $\mathrm{Ly} 6 \mathrm{G}^{+} \mathrm{c}-\mathrm{Kit}^{+}$blood cells: mean $11.1 \%$ in $\mathrm{TTP}^{\mathrm{fl} / \mathrm{fl}}$ vs. $6.1 \%$ in $\mathrm{TTP}^{\Delta \mathrm{M}}$ mice) (Figure 2, D and E). However, these modestly increased numbers of immature neutrophils in infected $\mathrm{TTP}^{\mathrm{fl} / \mathrm{fl}}$ mice did not result in changes in total neutrophil numbers (Figure 2A). Importantly, these data show that enhanced neutrophil infiltration in the skin of infected $\mathrm{TTP}^{\Delta \mathrm{M}}$ mice was not caused by increased granulopoiesis or numbers of immature neutrophils in the absence of TTP.

Together, the data demonstrate that myeloid lineage-specific ablation of TTP does not alter the steady-state reservoir of monocytes, macrophages, or neutrophils, nor does it alter bone marrow or circulating monocyte and neutrophil pools upon microbial challenge. These findings indicate that the ability of $\mathrm{TTP}^{\triangle \mathrm{M}}$ mice to augment immunity against $S$. pyogenes (Figure 1) results from enhanced defense at the site of infection.
TTP controls neutrophil but not monocyte accumulation. To examine the contribution of monocytes and neutrophils to the augmented local inflammatory response in $\mathrm{TTP}^{\Delta \mathrm{M}}$ mice, we analyzed myeloid subsets in a peritonitis model. Peritonitis was evoked by i.p. injection of heat-killed (HK) S. pyogenes, Staphylococcus aureus (both gram positive), or $E$. coli (gram negative), followed by collection of elicited cells 16 hours after challenge. HK bacteria were used in order to prevent systemic infection. Peritoneal exudates from $\mathrm{TTP}^{\Delta \mathrm{M}}$ mice challenged with HK $S$. pyogenes contained almost twice as many cells as the exudates from $\mathrm{TTP}^{\mathrm{f} / \mathrm{fl}}$ mice $\left(3.0 \pm 0.3 \times 10^{7}\right.$ vs. $1.6 \pm 0.2 \times 10^{7}$ ) (Figure $\left.3 \mathrm{~A}\right)$. We observed similar differences in peritoneal exudates from mice challenged with HK $S$. aureus or $E$. coli (Figure 3A). Peritoneal exudates obtained from $\mathrm{TTP}^{\Delta \mathrm{M}}$ animals had higher percentages of neutrophils $\left(\mathrm{Ly} 6 \mathrm{G}^{+} \mathrm{Ly} 6 \mathrm{C}^{+}\right)$when compared with those from $\mathrm{TTP}^{\mathrm{f} / \mathrm{fl}}$ controls (Figure $3 \mathrm{~B})$. Analysis of the absolute numbers of $\mathrm{HK}$ S. pyogenes-elicited cells revealed a 2 -fold higher infiltration of neutrophils into the peritoneum of $\mathrm{TTP}^{\Delta \mathrm{M}}$ mice compared with that observed in $\mathrm{TTP}^{\mathrm{f} / \mathrm{fl}}$ animals $\left(1.9 \pm 0.2 \times 10^{7}\right.$ and $0.8 \pm 0.1 \times 10^{7}$ neutrophils, respectively) (Figure 3C).We detected similar differences in HK S. aureus- and E. coli-challenged mice (Figure 3C). In contrast, the absolute numbers of monocytes were not significantly different between $\mathrm{TTP}^{\Delta \mathrm{M}}$ and $\mathrm{TTP}^{\mathrm{f} / \mathrm{ll}}$ animals, regardless of the pathogen used (Figure 3C). Consistently, the different neutrophil infiltration was no longer observed 48 hours after challenge, i.e., after the shift from neutrophilic to macrophagic inflammation (Supplemental Figure 3). These results demonstrate that $\mathrm{TTP}^{\Delta \mathrm{M}}$ mice have a greater capability of accumulating neutrophils in inflammatory foci than do $\mathrm{TTP}^{\mathrm{f} / \mathrm{fl}}$ controls. Interestingly, the infiltration of monocytes was not significantly regulated by TTP under these conditions. Thus, TTP is specifically restricting the neutrophil population at the site of infection.

TTP controls antimicrobial reprogramming of the neutrophil transcriptome. The enhanced accumulation of infiltrating neutrophils, but not monocytes, in $\mathrm{TTP}^{\Delta \mathrm{M}}$ mice (Figure 3) raised the question about the specific effects of TTP on the neutrophil transcriptome. To this end, we performed RNA-sequencing (RNA-seq) experiments using LPS-stimulated peritoneal neutrophils $\left(>95 \% \mathrm{Ly}^{6} \mathrm{G}^{+}\right)$isolated from $\mathrm{TTP}^{\Delta \mathrm{M}}$ and $\mathrm{TTP}^{\mathrm{f} / \mathrm{l} / \mathrm{l}}$ mice. Peritoneal neutrophils were elicited following a standard casein protocol (32). After isolation, neutrophils were stimulated using LPS, i.e., a stimulus utilized in reported transcriptome-wide studies of TTP function in other myeloid cells (12, 33-36). As expected, treatment of peritoneal neutrophils with LPS, lipoteichoic acid (LTA), or HK S. pyogenes increased TTP expression 
A
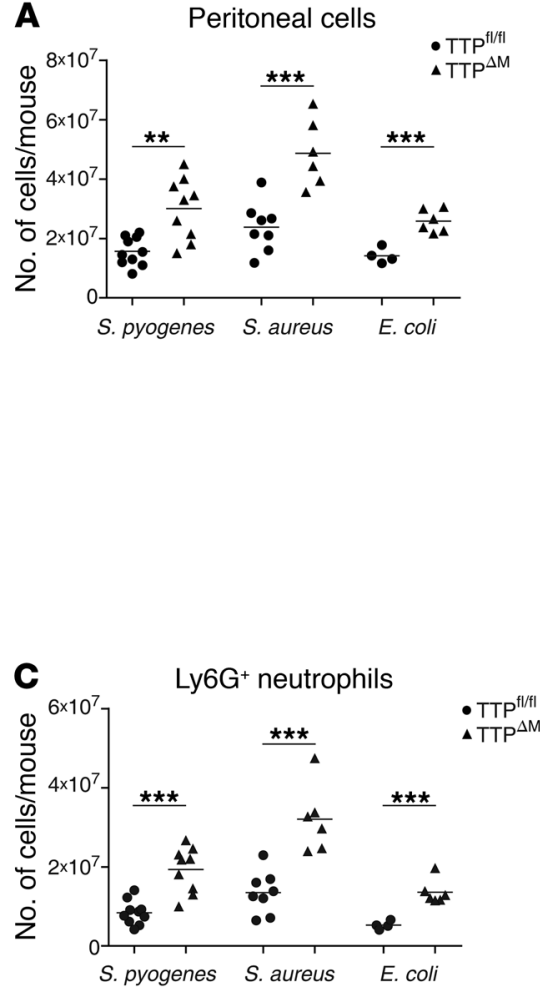

B
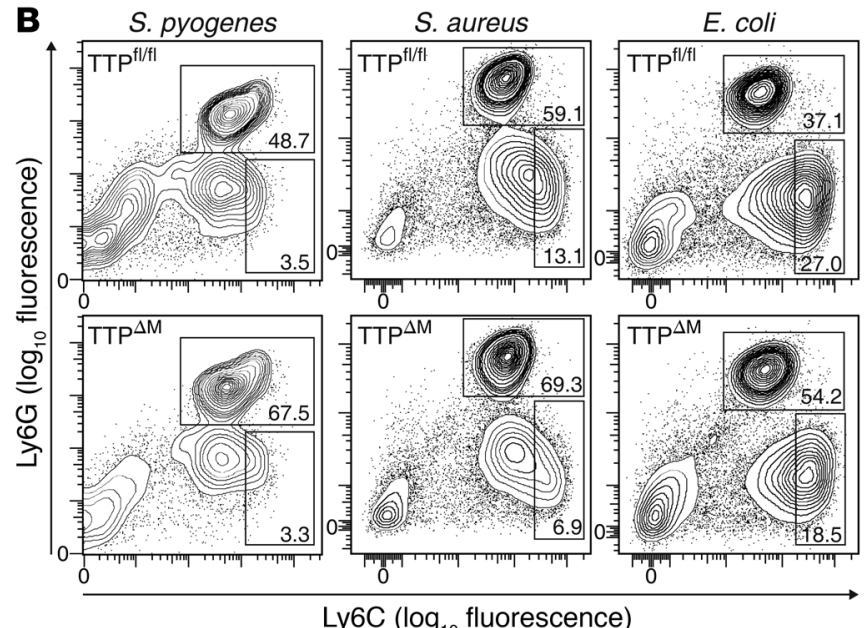

Ly6C ( $\log _{10}$ fluorescence)

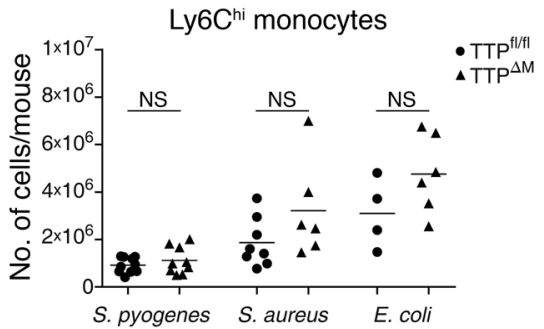

Figure 3. TTP ablation enhances neutrophil but not monocyte accumulation at the site of inflammation. (A) Mice were injected i.p. with $2 \times 10^{8} \mathrm{CFU}$ HK S. pyogenes ( $n=10 \mathrm{TTP}^{\mathrm{fl} / \mathrm{fl}}$ and $n=9 \mathrm{TTP}^{\Delta \mathrm{M}}$ mice) or HK S. aureus $\left(n=8 \mathrm{TTP}^{\mathrm{fl} / \mathrm{fl}}\right.$ and $n=6 \mathrm{TTP}^{\Delta \mathrm{M}}$ mice), or $2 \times 10^{6} \mathrm{CFU}$ HK E. coli $\left(n=4 \mathrm{TTP}^{\mathrm{fl} / \mathrm{fl}}\right.$ and $n=6$ TTP $\mathrm{TP}^{\Delta \mathrm{M}}$ mice) 16 hours prior to cell collection. Dot plot shows total viable peritoneal cells. (B and C) Mice were challenged, and peritoneal cells were collected as in A. Representative flow plots (B) and total numbers (C) of neutrophils (Ly6G+Ly6C+) and inflammatory monocytes (Ly6C-Ly6Chi) obtained from challenged mice. Numbers in B represent the percentages of the outlined cell populations. Error bars indicate the mean. ${ }^{* *} P<0.01$ and ${ }^{* * *} P<0.001$, by unpaired Student's $t$ test.

in $\mathrm{TTP}^{\mathrm{f} / \mathrm{fl}}$ neutrophils, whereas no expression was detected in $\mathrm{TTP}^{\Delta \mathrm{M}}$ neutrophils (Supplemental Figure 4A). Consistently, we found that expression of the TTP targets Tnf and Cxcl 2 in response to LPS, LTA, or HK S. pyogenes was higher in $\mathrm{TTP}^{\Delta \mathrm{M}}$ and $\mathrm{TTP}^{\mathrm{f} / \mathrm{fl}}$ neutrophils (Supplemental Figure 4B). The differences in Tnf and Cxcl 2 mRNA levels between $\mathrm{TTP}^{\Delta \mathrm{M}}$ and $\mathrm{TTP}^{\mathrm{fl} / \mathrm{fl}}$ neutrophils were greater at the 4-hour time point than at earlier time points (Supplemental Figure 4B), in agreement with the finding of increasing mRNA-destabilizing activity of TTP during the resolution phase of inflammation in macrophages $(35,36)$. Thus, the 4 -hour time point was selected for RNAseq analysis. Quantitation of independent triplicates revealed that the expression of 3,472 genes was significantly different between $\mathrm{TTP}^{\Delta \mathrm{M}}$ and $\mathrm{TTP}^{\mathrm{f} / \mathrm{fl}}$ neutrophils (Figure $4 \mathrm{~A}$ and Supplemental Table 1). We found that several TTP targets previously characterized in macrophages $(12,16)$ were elevated in $\mathrm{TTP}^{\Delta \mathrm{M}}$ neutrophils (e.g., Tnf and $\mathrm{CxCl2}$ ) (Figure 4A and Supplemental Table 1), which was consistent with their targeting for degradation in both macrophages and neutrophils. However, some other previously described targets (e.g., Il6 and Csf2) (Figure 4A and Supplemental Table 1) were decreased in $\mathrm{TTP}^{\triangle \mathrm{M}}$ neutrophils. As TTP prefers AREs in $3^{\prime}$-UTRs for binding (36), we asked whether TTP targets the canonical AREs (i.e., AUUUA pentamers) in neutrophils as well. Analysis of the AUUUA pentamer frequency in the $3^{\prime}$-UTRs of genes upregulated in $\mathrm{TTP}^{\triangle \mathrm{M}}$ neutrophils revealed that the magnitude (i.e., fold change) of upregulation in $\mathrm{TTP}^{\Delta \mathrm{M}}$ versus $\mathrm{TTP}^{\mathrm{f} / \mathrm{fl}}$ neutrophils was positively correlated with the number of AREs in the corresponding mRNA (Spearman's correlation, $0.25 ; P<2.2 \times 10^{-16}$ ) (Figure $4 \mathrm{~B}$ ).

These data showed that TTP targeted the canonical AUUUA pentamers in neutrophils. However, the differences between neutrophils and macrophages in the regulation of, for example, Il6 mRNA by TTP prompted us to compare the transcriptome-wide differential expression analysis of TTP-deficient and -proficient neutrophils with a similar analysis we recently reported for macrophages treated for 3 hours with LPS (36). Strikingly, our comparison of differently expressed genes revealed no correlation between neutrophils and macrophages (Pearson's correlation $R=-0.005$ ) (Figure 4C). Examples of similarly regulated genes include $T n f$ and $C x c l 2$, which show increased expression in TTP-deficient cells (Figure 4C). In contrast, Nos 2 and $I l 1 b$ were elevated in TTP-deficient neutrophils but not macrophages, whereas Il6 was reduced in TTP-deficient neutrophils, but was increased in TTP-deficient macrophages (Figure 4C). These data indicate that TTP-dependent mRNA decay has distinct effects on the transcriptome in neutrophils and macrophages.

Immunostimulation of neutrophils causes a marked increase in the expression of genes that are functionally classified by gene ontology (GO) into biological process categories including cell motility, cytoskeletal rearrangement, apoptosis, regulation of cell proliferation, and events involving the Rho family of small GTPases (37). These processes are involved in neutrophil extravasation and accumulation at the site of infection as well as in the 

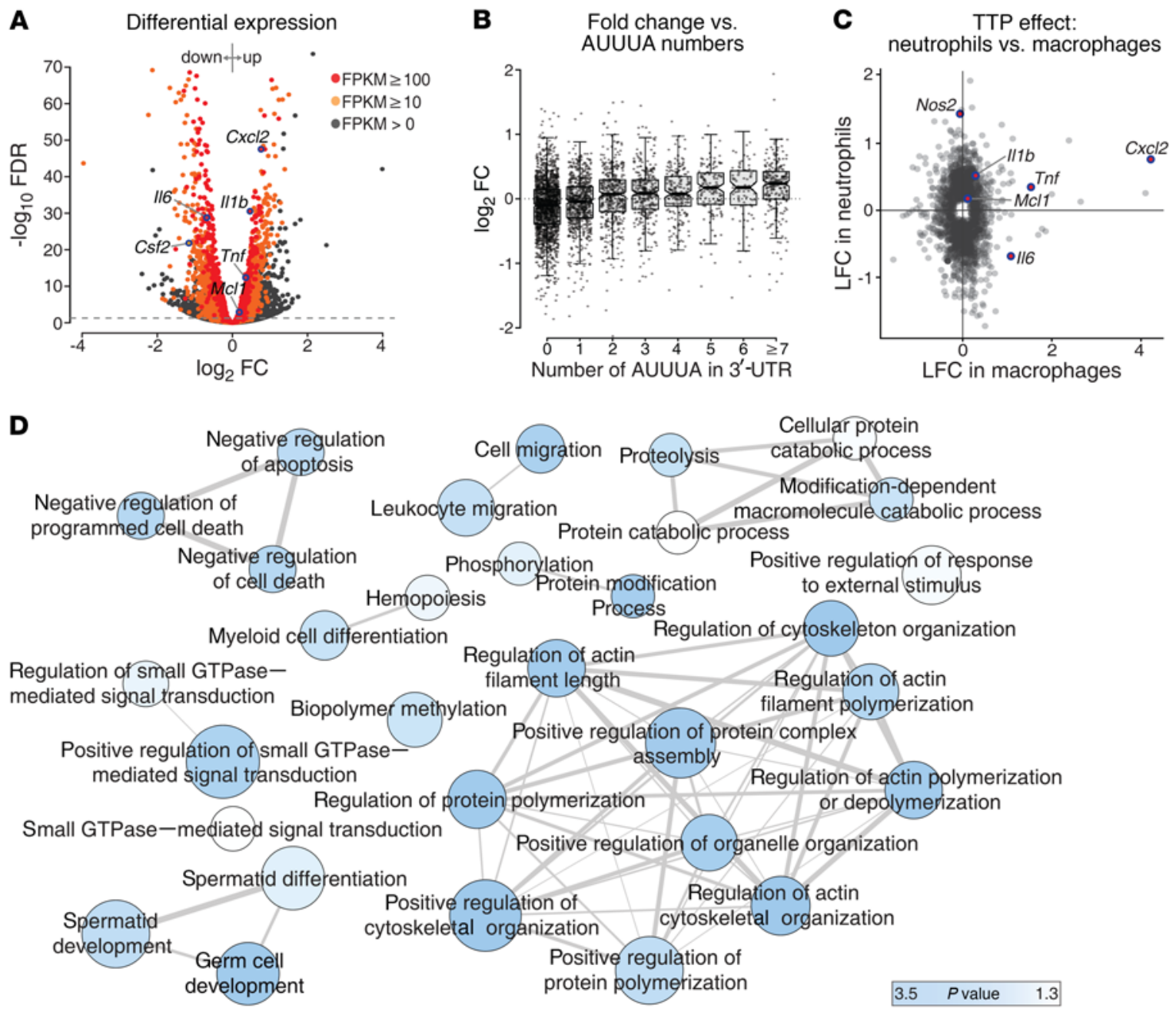

Figure 4. TTP controls antimicrobial activation of the neutrophil transcriptome. Peritoneal neutrophils (>95\% Ly6G ${ }^{+}$) from TTP ${ }^{\Delta M}$ and TTP ${ }^{f / / f l}$ mice were stimulated with $10 \mathrm{ng} / \mathrm{ml}$ LPS for 4 hours, and differential expression was analyzed. (A) Volcano plot showing the statistical significance of different expression levels (FDR) against fold changes (FC) of expression between TTP ${ }^{\Delta M}$ and TTP ${ }^{\mathrm{fl} / \mathrm{fl}}$ neutrophils. Dots represent genes color-coded according to expression levels (FPKM). Horizontal dashed line indicates an FDR of 0.05. Cxcl2, I/1b, Csf2, I/6, Mcl1, and Tnf represent examples of differently expressed genes. (B) Boxand-whisker plots show the FC in gene expression between TTP ${ }^{\Delta M}$ and TTP ${ }^{f l / f l}$ neutrophils dependent on AUUUA numbers in $3^{\prime}-U T R s$ (FPKM $\left.>10\right)$. (C) Correlation plots for TTP-dependent differential gene expression in neutrophils and macrophages. Differential gene expression in TTP $\mathrm{P}^{\Delta \mathrm{M}}$ and TTP $\mathrm{P}^{\mathrm{fl} / \mathrm{fl}}$ neutrophils ( $y$ axis) and TTP ${ }^{\Delta M}$ and TTP ${ }^{f / f l}$ macrophages ( $x$ axis) after LPS treatment is shown as the LFC. No correlation between differential gene expression in neutrophils or macrophages was observed (Pearson's $R=-0.005 ; 95 \% \mathrm{Cl},-0.052$ to $0.040 ; P=0.8242$ ). (D) CO enrichment in the category "biological process" for significantly upregulated genes in TTP ${ }^{\Delta M}$ neutrophils $(F P K M>10)$. Edges connect $\mathrm{GO}$ terms sharing multiple genes (Jaccard distance $\left.\leq 0.8\right)$; line width indicates the Jaccard distance. Circle size shows term enrichment; circle color shows the $P$ value (from blue to white, $P$ value is shown as - log $_{10}$ ). Note the clustering of terms for the negative regulation of programmed cell death (top left), cell migration (top middle), and cytoskeletal rearrangement (bottom right).

acquisition of antimicrobial properties $(1,2)$. Mutations in genes facilitating extravasation (e.g., integrins) and cytoskeletal remodeling or mutations promoting neutrophil cell death are causative in patients suffering from neutrophil defects $(1,2)$. GO enrichment analysis of genes elevated in $\mathrm{TTP}^{\Delta \mathrm{M}}$ neutrophils revealed that TTP regulates processes important for neutrophil function: genes associated with cell migration, cytoskeletal rearrangement, and negative regulation of programmed cell death were significantly overrepresented among genes more highly expressed in $\mathrm{TTP}^{\Delta \mathrm{M}}$ neutrophils (Figure 4D and Supplemental Table 2).

In conclusion, TTP shapes the transcriptome of activated neutrophils by controlling the expression of mRNAs associated with key aspects of neutrophil responses. The impact of TTP on the neutrophil transcriptome differs from that of macrophages, implying the existence of distinct properties of TTP-mediated mRNA decay in these 2 myeloid cell types.

TTP promotes apoptosis of infiltrating neutrophils. The accumulation of neutrophils, which constitute the most abundant immune cell population at the site of primary infection, is significantly augmented in the absence of TTP (Figure 3). The differential gene expression analyses revealed, among other findings, a higher expression of negative regulators of cell death in $\mathrm{TTP}^{\Delta \mathrm{M}}$ neutrophils (Figure 4). This suggested that TTP might negatively regulate the lifespan of neutrophils and thereby restrict their numbers at the site of infection. To answer this question, we examined apoptosis of peritoneal neutrophils elicited using HK S. pyogenes. Our analysis of annexin V staining revealed that neutrophils from $\mathrm{TTP}^{\mathrm{fl} / \mathrm{ll}}$ mice had an approximately 2 -fold higher $(P<0.001)$ per- 

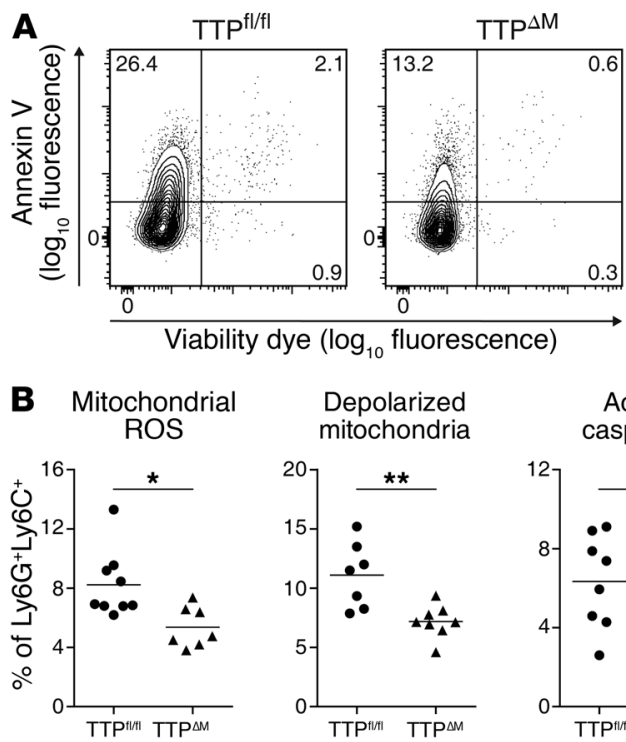

Depolarized mitochondria

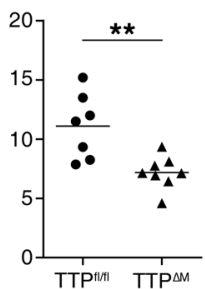

Active caspase-3

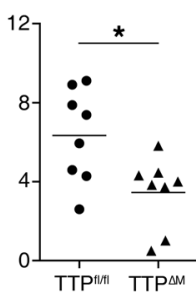

Annexin $\mathrm{V}^{+}$neutrophils

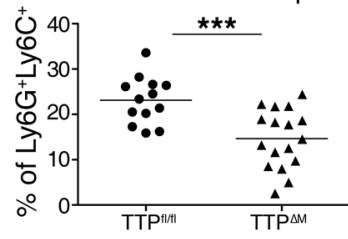

C Efferocytosed neutrophils

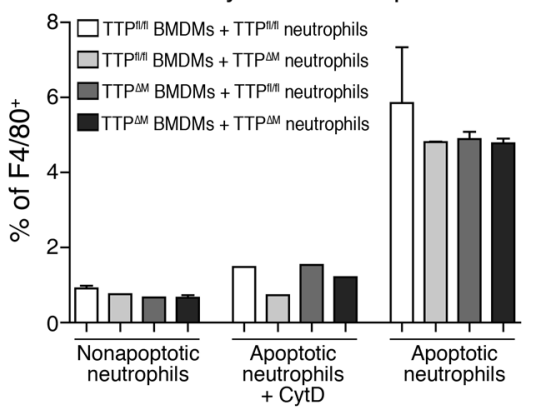

D

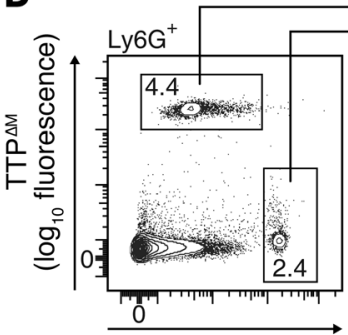

TTP $\stackrel{\text { tiffil }}{\left(\log _{10} \text { fluorescence }\right)}$

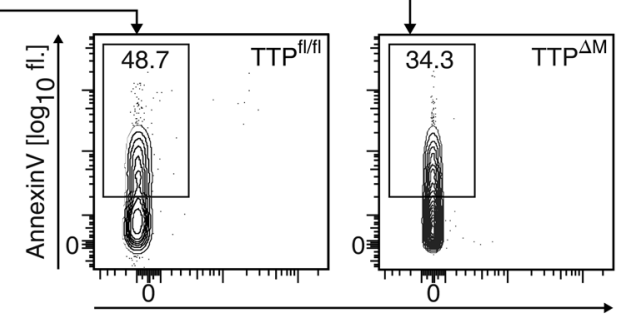

Viability dye ( $\log _{10}$ fluorescence)

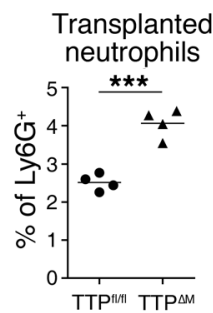

Annexin $\mathrm{V}^{+}$ neutrophils
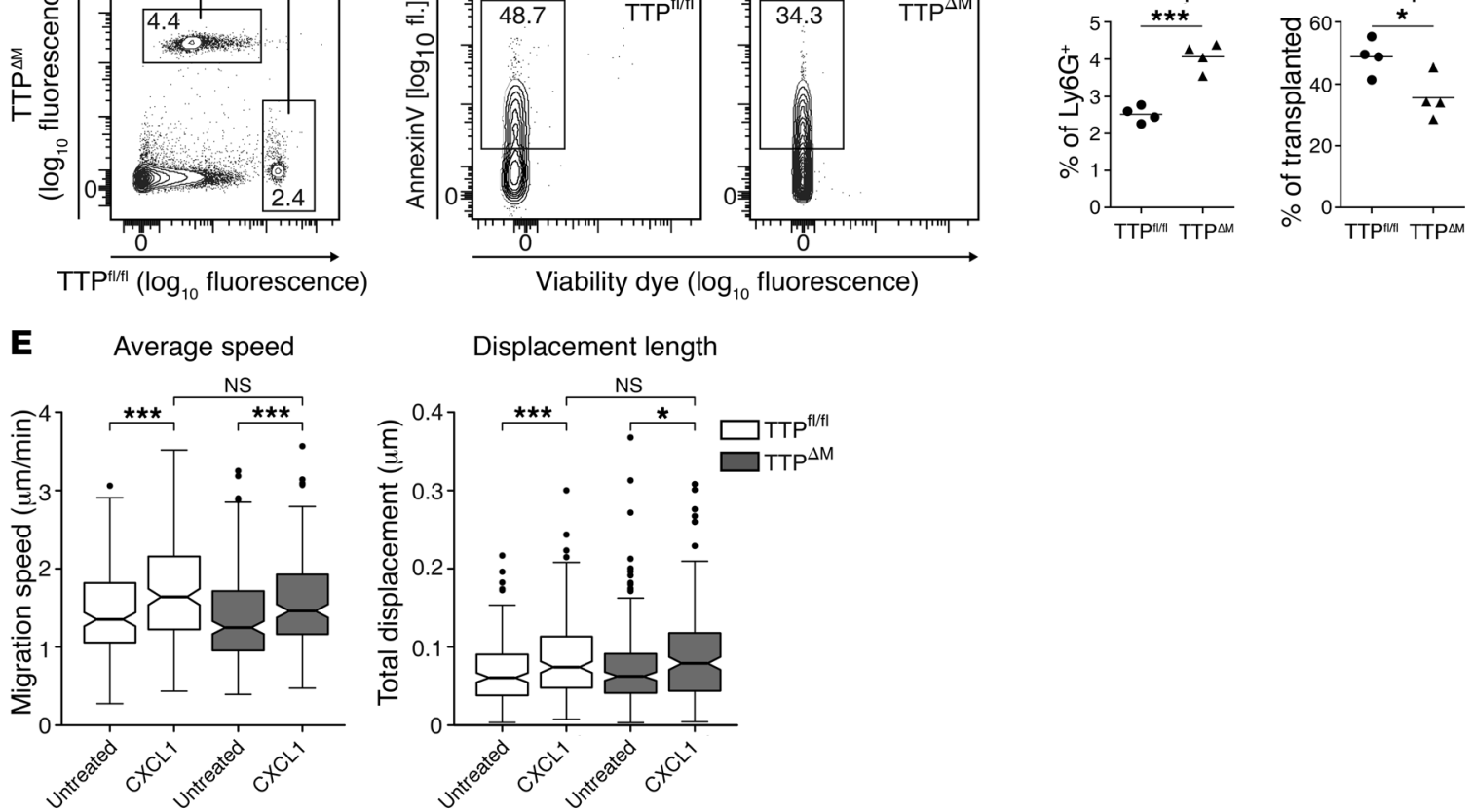

Displacement length

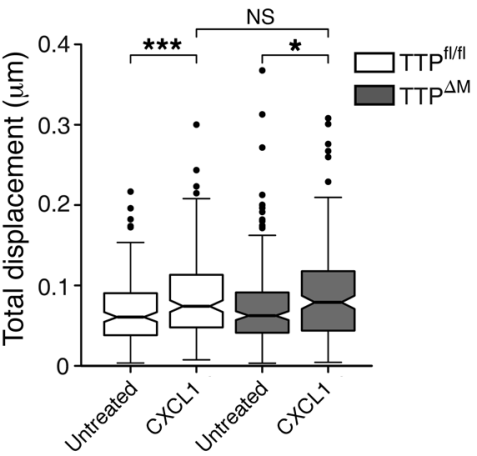

Figure 5. TTP promotes neutrophil apoptosis in a cell-intrinsic manner. (A) Apoptosis was analyzed in peritoneal neutrophils elicited using $2 \times 10^{8} \mathrm{CFU} H \mathrm{~K}$ S. pyogenes 16 hours prior to flushing of the peritoneal cavity. Representative flow plots show annexin $\mathrm{V}$ and live/dead staining of Ly6 $\mathrm{C}^{+} \mathrm{Ly} 6 \mathrm{C}^{+}$neutrophils. The threshold for annexin $\mathrm{V}^{+}$signal was based on fluorescence minus one (FMO) staining without annexin V. Numbers represent the percentage of cells in the respective quadrants. Dot plot for 3 pooled experiments $\left(n=13\right.$ TTP $\left.^{\mathrm{fl} / \mathrm{fl}} ; n=17 \mathrm{TTP}^{\Delta \mathrm{M}}\right)$ depicts the percentages of early apoptotic neutrophils (living annexin $\left.\mathrm{V}^{+}\right)$. (B) Neutrophils, elicited as in A, were analyzed for mitochondrial superoxide production $(n=9$ TTP fl/ff; $n=8$ TTP $4 \mathrm{M})$, polarization of mitochondrial membrane ( $\left.n=7 \mathrm{TTP}^{\mathrm{fl} / f \mathrm{f}} ; n=8 \mathrm{TTP}^{\Delta \mathrm{M}}\right)$, and active caspase-3 $\left(n=8 \mathrm{TTP}^{\mathrm{fl} / \mathrm{fl} ;} n=8 \mathrm{TTP}^{\Delta \mathrm{M}}\right)$. (C) TTP $\mathrm{TP}^{\mathrm{f} / \mathrm{fl}}$ and TTP $\mathrm{TT}^{\Delta \mathrm{M}}$ BMDMs were incubated with pHrodo-stained nonapoptotic or apoptotic TTP ${ }^{f / f 1}$ and TTP ${ }^{\Delta M}$ neutrophils at a BMDM/neutrophil ratio of 1:2. CytD-pretreated BMDMs served as a control for adherent neutrophils. Bar graph shows the percentage of pHrodo F4/80+ BMDMs $(n=3)$. Error bars indicate the mean \pm SD. (D) Casein-elicited neutrophils from TTPf//fl and TTP ${ }^{\Delta M}$ mice were differently labeled, mixed at a 1:1 ratio, and injected i.p. into 4 recipient TTPfl/fl animals, in which peritonitis was induced 3 hours earlier using HK S. pyogenes. Representative flow plots 18 hours after transplantation and dot plots show retrieved transplanted neutrophils and the percentage of annexin $\mathrm{V}^{+}$cells therein. (E) Neutrophil migration through a collagen matrix was followed by live imaging. Box plots show the average speed and displacement length of TTP $\mathrm{fl}^{\prime / f}$ and TTP ${ }^{\Delta \mathrm{M}}$ neutrophils in untreated conditions or in a CXCL1 gradient. Error bars in $\mathbf{A}, \mathbf{B}$, and $\mathbf{D}$ represent the mean. Statistical analysis was determined by unpaired $(\mathbf{A}-\mathbf{D})$ or paired $(\mathbf{E})$ Student's $t$ test. ${ }^{*} P<0.05,{ }^{* *} P<0.01$, and ${ }^{* * *} P<0.001$. 
A Mcl1 mRNA expression
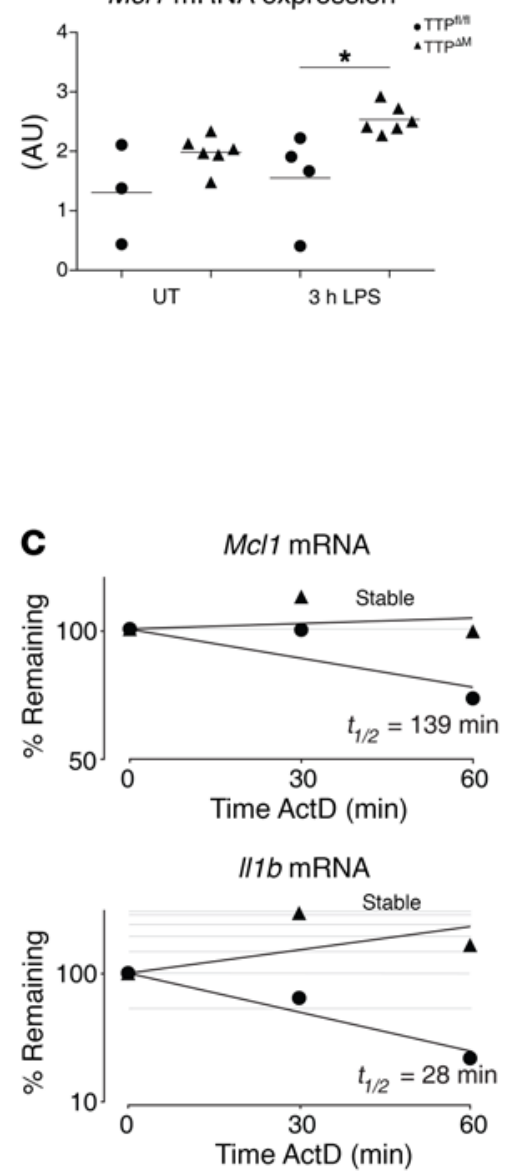

D

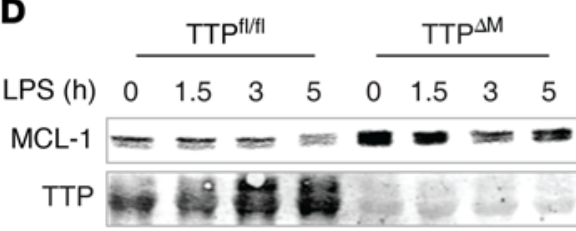

Tubulin $-\ldots$

Figure 6. TTP binds and destabilizes Mcl1 mRNA and decreases MCL-1 protein abundance. (A) Mcl1 transcript levels in elicited peritoneal cells $\left(n=4 \mathrm{TTP}^{\mathrm{fl} / f l} ; n=6 \mathrm{TTP}^{\Delta \mathrm{M}}\right)$ stimulated with LPS. Error bars represent the mean. ${ }^{*} P<0.05$, by unpaired Student's $t$ test. (B) PAR-CLIP analysis of TTP binding to Mcl1 transcript (ENSMUS00000037947) in BMDMs stimulated with $10 \mathrm{ng} / \mathrm{ml}$ LPS. Bars show the number of crosslink (CL) events between Mc/1 mRNA and TTP at nucleotide (color-coded) resolution. AUUUA pentamers and the position of pentamers conserved in mouse and human Mcl1/MCL1 are indicated. nt, nucleotide; TSS, transcription start site. (C) Peritoneal neutrophils $\left(5 \times 10^{6}\right)$ were stimulated with LPS for 3 hours, followed by mRNA decay assays. Plots show linear regression fitted in semi-logarithmic coordinates representing the percentage of remaining mRNA after transcriptional blockage (time point 0 ). Half-lives $\left(t_{1 / 2}\right)$ were calculated from the linear model. Hprt was used as a negative control. (D) Neutrophils were isolated and stimulated with LPS $(10 \mathrm{ng} / \mathrm{ml})$. Whole-cell extracts were prepared, and MCL-1 and TTP levels were determined by Western blotting. Tubulin was used as a loading control.

centage of apoptotic cells than did neutrophils from $\mathrm{TTP}^{\Delta \mathrm{M}}$ mice (Figure 5A). We observed similar differences for other indicators of apoptotic cell death, i.e., mitochondrial ROS production (38), mitochondrial membrane depolarization, and caspase-3 activation (39) (Figure 5B and Supplemental Figure 5A). The absolute numbers of apoptotic cells varied among the indicators because of the different sensitivity of the methods used.
Since the lower number of apoptotic $\mathrm{TTP}^{\Delta \mathrm{M}}$ neutrophils could be a result of enhanced clearance of these cells by macrophages, we assessed efferocytosis (i.e., phagocytosis) of apoptotic neutrophils by macrophages. $\mathrm{TTP}^{\Delta \mathrm{M}}$ and $\mathrm{TTP}^{\mathrm{fl} / \mathrm{fl}}$ neutrophils were treated with hydrogen peroxide and PMA, which rapidly induce complete apoptosis (40). After labeling with the $\mathrm{pH}$-sensitive dye pHrodo, apoptotic neutrophils were incubated with $\mathrm{TTP}^{\Delta \mathrm{M}}$ and $\mathrm{TTP}^{\mathrm{f} / \mathrm{fl}}$ bone marrow-derived macrophages (BMDMs), followed by determination of pHrodo-positive BMDMs. The numbers of BMDMs containing phagocytosed apoptotic neutrophils were similar in all 4 combinations (Figure $5 \mathrm{C}$ and Supplemental Figure 5B). The fluorescence of BMDMs was derived from phagocytosed rather than adherent neutrophils, as evidenced by the strong decrease in pHrodo-positive BMDMs upon blockage of phagocytosis by cytochalasin D (CytD) (Figure 5C). A phagocytosis assay using nonapoptotic neutrophils (i.e., neutrophils not treated with hydrogen peroxide and PMA) generated a low fluorescence signal that was in the range of that for CytD-treated BMDMs (Figure $5 \mathrm{C}$ ). These data indicated that enhanced clearance of apoptotic $\mathrm{TTP}^{\triangle \mathrm{M}}$ neutrophils by macrophages could not account for the lower frequency of apoptotic neutrophils in $\mathrm{TTP}^{\Delta \mathrm{M}}$ mice.

We then examined whether the decreased apoptosis of neutrophils isolated from $\mathrm{TTP}^{\Delta \mathrm{M}}$ mice was a cell-autonomous effect or whether it was dependent on other TTP-deficient cells. Equal numbers of differently labeled $\mathrm{TTP}^{\mathrm{fl} / \mathrm{fl}}$ and $\mathrm{TTP}^{\Delta \mathrm{M}}$ peritoneal neutrophils were mixed and transplanted into the peritoneum of $\mathrm{TTP}^{\mathrm{fl} / \mathrm{fl}}$ recipient mice that were subjected to peritonitis induction 3 hours before neutrophil injection. Analysis of transplanted neutrophils 18 hours after their injection showed that transplanted $\mathrm{TTP}^{\Delta \mathrm{M}}$ neutrophils were significantly more abundant than were transplanted $\mathrm{TTP}^{\mathrm{fl} / \mathrm{fl}}$ neutrophils: 4.1\% $\mathrm{TTP}^{\Delta \mathrm{M}}$ versus $2.5 \% \mathrm{TTP}^{\mathrm{fl} / \mathrm{fl}}$ of total neutrophils isolated from recipient mice (Figure 5D). Moreover, the transplanted $\mathrm{TTP}^{\Delta \mathrm{M}}$ neutrophils contained fewer apoptotic cells than did the transplanted $\mathrm{TTP}^{\mathrm{f} / \mathrm{fl}}$ neutrophils (35.6\% vs. $48.3 \%$ ) (Figure 5D). Thus, under the same external cues, $\mathrm{TTP}^{\Delta \mathrm{M}}$ neutrophils remained longer and had a lower apoptosis rate in the peritoneum than did $\mathrm{TTP}^{\mathrm{fl} / \mathrm{fl}}$ neutrophils. These results demonstrated that the higher peritoneal neutrophil numbers (Figure 3) and decreased neutrophil apoptosis (Figure 5, A and $\mathrm{B}$ ) in $\mathrm{TTP}^{\triangle \mathrm{M}}$ mice were largely caused by cell-autonomous mechanisms.

The neutrophil transplantation experiment (Figure 5D) indicated that the higher numbers of $\mathrm{TTP}^{\Delta \mathrm{M}}$ peritoneal neutrophils were predominantly a result of post-recruitment mechanisms. To provide more direct evidence for an insignificant role of TTP in neutrophil migration, we used video microscopy to monitor CXCL1-induced neutrophil migration. Peritoneal neutrophils from $\mathrm{TTP}^{\Delta \mathrm{M}}$ and $\mathrm{TTP}^{\mathrm{f} / \mathrm{fl}}$ mice were mounted onto a collagen 
A
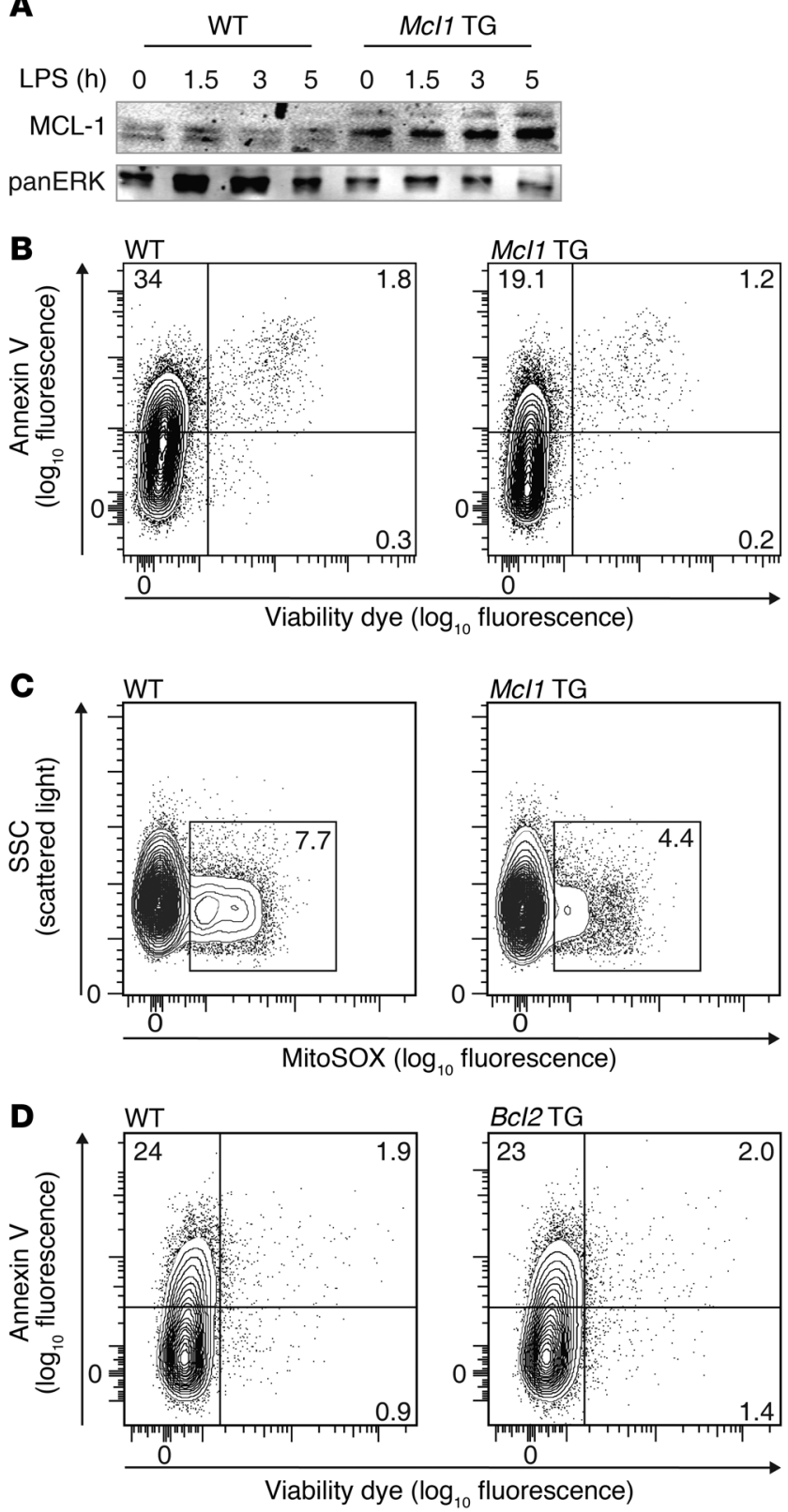

matrix and exposed to a CXCL1 gradient. Our analysis of trajectories revealed an increased migration of both $\mathrm{TTP}^{\mathrm{AM}}$ and $\mathrm{TTP}^{\mathrm{A} / \mathrm{I}}$ neutrophils upon their exposure to the chemotactic gradient (Figure 5E). Importantly, both the average speed and displacement length were comparable in $\mathrm{TTP}^{\triangle \mathrm{M}}$ and $\mathrm{TTP}^{\mathrm{A} / \mathrm{l}}$ neutrophils (Figure $5 E)$, demonstrating that neutrophil migration is not significantly regulated by TTP under these conditions.

In conclusion, TTP deficiency increases accumulation and decreases apoptosis of peritoneal neutrophils via cell-autonomous mechanisms. The enhanced peritoneal neutrophil accumulation observed in $\mathrm{TTP}^{\triangle \mathrm{M}}$ mice was not caused by increased neutrophil migration, implying that the underlying mechanism is a lower rate of $\mathrm{TTP}^{\triangle \mathrm{M}}$ neutrophil apoptosis.

TTP binds and destabilizes $M R N A$ of the antiapoptotic protein MCL-1. MCL-1 is a member of the B cell leukemia/lymphoma 2 (BCL-2) family of antiapoptotic proteins and was shown to promote
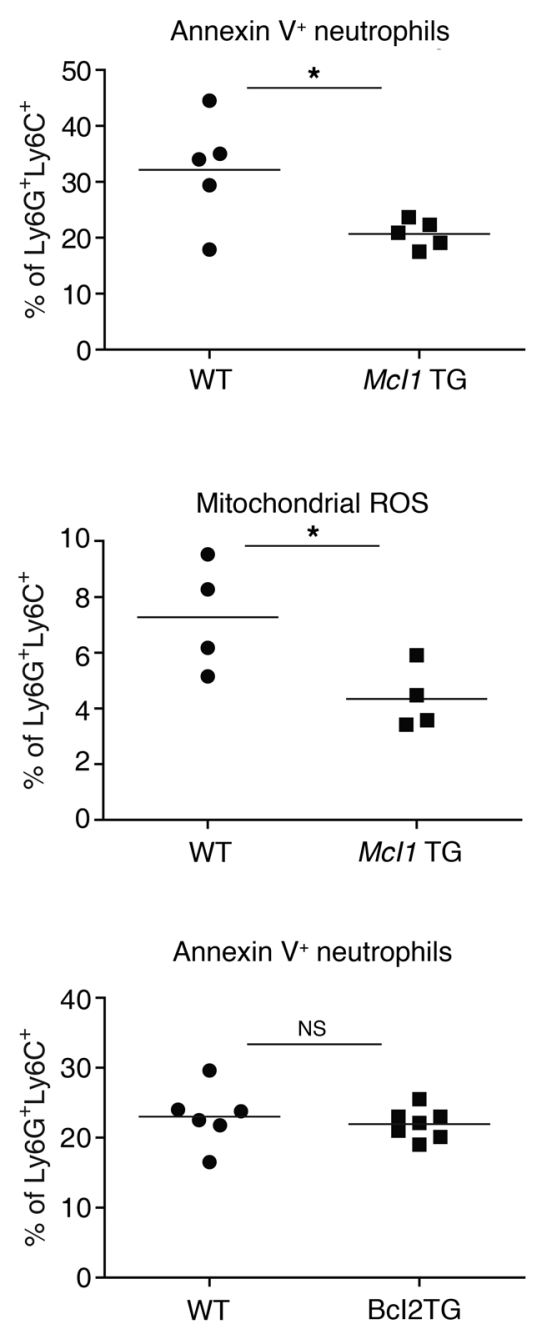

survival in a wide range of hematopoietic cells at different stages of development (41). MCL-1 plays a crucial and specific antiapoptotic role during the differentiation of neutrophils under homeostatic conditions, as revealed by myeloid-specific Mcl1-knockout mice, which spontaneously develop severe neutropenia but have normal macrophage numbers $(18,42)$. Our differential expression analysis revealed that $M c l 1 \mathrm{mRNA}$ was expressed at a significantly higher level in casein-elicited, LPS-stimulated TTP ${ }^{\triangle \mathrm{M}}$ neutrophils than was detected in neutrophils from TTP ${ }^{\mathrm{f} / \mathrm{fl}}$ littermate controls (Supplemental Table 1). To substantiate this finding, we examined Mcl1 expression using HK $S$. pyogenes-elicited neutrophils that were subsequently treated with LPS or left untreated. Stimulation with LPS was used, because it further increases both TTP levels and differences in TTP target gene expression (Supplemental Figure 4). We found that Mcl1 expression was consistently higher in neutrophils from $\mathrm{TTP}^{\triangle \mathrm{M}}$ mice than in neutrophils from $\mathrm{TTP}^{\mathrm{A} / \mathrm{I}}$ 
littermates prior to LPS treatment, and this difference increased after LPS treatment (Figure 6A). These data suggested that TTP promotes the apoptosis rate in $\mathrm{TTP}^{\mathrm{fl} / \mathrm{fl}}$ neutrophils by destabilizing Mcl1 mRNA. By searching our recently reported PAR-CLIPderived (photoactivatable ribonucleoside-enhanced crosslinking and IP-derived) high-resolution TTP-binding site atlas (http:// ttp-atlas.univie.ac.at/) in the macrophage transcriptome (36), we identified TTP binding in the Mcl1 3'-UTR at a position containing AREs conserved in the mouse and human genes (Figure 6B). We further confirmed TTP binding to Mcl1 mRNA by UV crosslinking and RNA-IP using BMDMs stimulated with LPS. Mcl1 mRNA was found to bind TTP to an extent similar to that seen with the known TTP targets Ccl4, Il6, and Illb mRNAs (Supplemental Figure 6A). We detected no binding for hypoxanthine guanine phosphoribosyl transferase (Hprt) mRNA, which served as a negative control.

To prove a direct effect of TTP on Mcl1 mRNA stability in neutrophils, we used mRNA decay assays with purified peritoneal neutrophils. We used the p38 MAPK inhibitor SB203580 to obtain robust TTP-mediated mRNA destabilization, as described previously (12). Neutrophils were stimulated with LPS for 3 hours, and transcription was then stopped by actinomycin $\mathrm{D}$ (ActD) treatment. The remnant $M c l 1 \mathrm{mRNA}$ was more stable in $\mathrm{TTP}^{\Delta \mathrm{M}}$ neutrophils than in $\mathrm{TTP}^{\mathrm{f} / \mathrm{fl}}$ cells (stable vs. half-life of 139 min, respectively) (Figure 6C). Cxcl2 and Il1b mRNAs, i.e., TTP targets previously characterized in BMDMs (12), were destabilized by TTP (Figure 6C). Moreover, Western blot analysis revealed that MCL-1 protein amounts were higher in $\mathrm{TTP}^{\triangle \mathrm{M}}$ neutrophils than in $\mathrm{TTP}^{\mathrm{f} / \mathrm{fl}}$ neutrophils (Figure 6D and Supplemental Figure 6B). Similar to Mcl1 mRNA analysis (Figure 6A), MCL-1 protein was already higher in $\mathrm{TTP}^{\Delta \mathrm{M}}$ neutrophils prior to LPS treatment, which is consistent with substantial TTP protein levels under these conditions (Figure 6D). The differences in MCL-1 protein levels between $\mathrm{TTP}^{\Delta \mathrm{M}}$ and $\mathrm{TTP}^{\mathrm{f} / \mathrm{fl}}$ neutrophils increased slightly throughout the LPS treatment (Supplemental Figure 6B), showing that higher TTP levels could further decrease, but not completely abrogate, MCL-1 amounts. This might result from MCL-1 protein stabilization under these conditions, as MCL-1 expression is known to be significantly regulated at the level of protein stability $(43,44)$. These data show that TTP markedly reduces MCL-1 protein levels in peritoneal neutrophils and substantiate the hypothesis that TTP promotes neutrophil apoptosis by reducing $M c l 1$ expression. The substantial expression of TTP in elicited peritoneal neutrophils (i.e., without LPS stimulation) is in agreement with the finding of an inflammatory milieu in the peritoneal cavity following administration of the eliciting agent (e.g., casein or HK S.pyogenes).

To corroborate the antiapoptotic effect of increased MCL-1 levels in $\mathrm{TTP}^{\Delta \mathrm{M}}$ neutrophils, we used mice overexpressing MCL-1 in hematopoietic cells (referred to hereafter as Mcl1 TG mice), which were reported to exhibit decreased neutrophil apoptosis upon exposure to environmental stress (45). The extent of MCL-1 overexpression was similar in both $\mathrm{TTP}^{\triangle \mathrm{M}}$ and $\mathrm{Mcl} 1 \mathrm{TG}$ neutrophils when compared with expression levels in corresponding controls (Figure 6D, Figure 7A, and Supplemental Figure 6, B and C). Importantly, HK S. pyogenes-elicited cells from Mcl1 TG mice had approximately $40 \%$ fewer $(P<0.05)$ apoptotic neutrophils than did cells from control animals (Figure 7, B and C). Thus, neutrophils from Mcl1 TG mice were protected against apoptosis after bacterial challenge to an extent similar to that observed in neutrophils lacking TTP. By using Bcl2 TG animals, we could show that overexpression of $B c l 2$, which inhibits apoptosis in several hematopoietic lineages (46), did not regulate the apoptosis of HK S. pyogenes-elicited neutrophils (Figure 7D), confirming the specific effect of $M c l 1$ on neutrophil apoptosis during infection. Expression levels of other antiapoptotic $B c l 2$ family members, including Bcl2l11, Bcl2l13, Bcl2a1a, Bcl2a1b, $B c l 2 a 1 c, B c l 2 a 1 d$, and $B c l 2 l 2$, were not elevated in TTP-deficient neutrophils (Supplemental Table 1), further strengthening our hypothesis that the increased Mcl1 expression in TTP-deficient neutrophils promoted their survival.

In summary, these data indicate a causal role of TTP deficiency in high Mcl1 expression and Mcl1-dependent survival of immunostimulated neutrophils. These results, together with normal neutrophil numbers in unchallenged $\mathrm{TTP}^{\Delta \mathrm{M}}$ mice (Figure 2), establish the idea that TTP-mediated destabilization of Mcl1 mRNA facilitates apoptosis in activated pathogen-engaged neutrophils, whereas the survival of neutrophils under homeostatic conditions is not regulated by TTP.

\section{Discussion}

This study reveals that the duration of neutrophil engagement with pathogens is temporarily limited by the RNA-binding protein TTP. The effects of TTP on the neutrophil transcriptome differ from those described in macrophages and demonstrate unexpected cell type-specific properties of mRNA stability control. TTP regulates neutrophils involved in antimicrobial defense, but not the resting neutrophil pool, by promoting apoptosis of pathogen-engaged neutrophils and restricting expression of the antiapoptotic $M c l 1$ gene. The increased pathogen engagement of TTP-deficient neutrophils results in a more efficient bacterial clearance and improved immunity against invasive bacterial infection of the soft tissue. Our data support the previous notion that apoptosis is the key mechanism for restricting activated neutrophils (3).

The present work shows that mRNA destabilization by TTP has a broader impact on the transcriptome of neutrophils than on that of macrophages, in which mostly cytokine and chemokine mRNAs were found to be regulated (36). This difference might be caused by thus-far insufficiently explored cell type-specific aspects of mRNA destabilization by TTP. Alternatively, a more significant effect of TTP on the transcriptome might be a characteristic property of very short-lived cells like neutrophils. Our observations that mRNAs restricted by TTP in macrophages (e.g., Il6) are not regulated in this way in neutrophils, whereas other mRNAs (e.g., Nos2) are controlled in neutrophils but not macrophages, suggest that TTP target selection might indeed be significantly cell-type dependent. Transcriptome-wide studies in other immune cells are needed to substantiate this hypothesis. Such studies will also help to explore the hypothesis that the transcriptome of short-lived cells is in general more strongly regulated at the posttranscriptional level. This hypothesis is conceivable, since mRNA decay is likely to have a robust impact under conditions of diminished RNA synthesis resulting from nucleotide depletion in dying cells (47). Of importance for the fate of short-lived cells is the regulation of celldeath genes. In agreement with this, we found negative regulators of apoptosis to be enriched among the TTP targets in neutrophils. 
Although TTP deficiency reduces neutrophil apoptosis, the neutrophil homeostasis under steady-state conditions is not impaired, implying that TTP regulates neutrophil survival upon infection, but not during homeostatic differentiation or circulation.

We propose that the lower rate of apoptosis of activated $\mathrm{TTP}^{\Delta \mathrm{M}}$ neutrophils is causally related to the elevated levels of the antiapoptotic protein MCL-1, whose mRNA is destabilized in a TTPdependent way in neutrophils. $M c l 1$ is a key neutrophil survival factor, and the absence of $\mathrm{Mcl} 1$ reduces circulating neutrophils by $80 \%$ (42). In contrast, $M c l 1$ ablation has no effect on macrophages (42). The antiapoptotic function of $M c l 1$ is required throughout the neutrophil life cycle, since $M c l 1$ deficiency impairs both steadystate neutrophils and neutrophils exposed to environmental stress $(42,48)$. A key unresolved question was how the Mcl1-controlled neutrophil lifespan is adjusted to match the requirements for both sufficient neutrophil supply and a balanced duration of neutrophil deployment. Our findings showing that TTP selectively controls the cellularity of infiltrating neutrophils and limits $\mathrm{Mcl} 1$ expression in these cells indicate that TTP acts as a timer of $\mathrm{Mcl} 1$ activity upon neutrophil engagement with pathogens. The involvement of TTP in the regulation of $\mathrm{Mcl1}$ is specific, since the expression of other $B c l 2$ family members, including the neutrophil-relevant $B c l 2 a 1 a$ $(49,50)$, is not augmented in TTP-deficient neutrophils. In further support of the selective role of the TTP/Mcl1 axis in apoptosis of pathogen-engaged neutrophils, we did not observe an effect of $\mathrm{Bcl} 2$ overexpression on the apoptosis of $S$.pyogenes-elicited neutrophils.

Cutaneous infections by streptococci and staphylococci in humans can rapidly progress to severe systemic infections that are accompanied by high mortality caused by toxic shock (51). It has remained unclear whether the transition from a localized to a disseminated and life-threatening disease is the consequence of an insufficient immune response at the infection site or, on the contrary, of local hyperinflammation and tissue damage that facilitate spreading of the bacteria $(22,51)$. Our findings support the concept that a strong immune response at the primary site of infection, as observed in $\mathrm{TTP}^{\Delta \mathrm{M}}$ mice, helps to contain bacteria, thereby preventing systemic infection and inflammation. Although the increased numbers of infiltrating neutrophils in the $\mathrm{TTP}^{\Delta \mathrm{M}}$ mice are associated with severe tissue damage in the area of infection, the overall consequences are beneficial to the host. The systemic overspill of the local hyperinflammation might be counteracted by homeostatic mechanisms that prevail in distant organs in the absence of bacterial dissemination. Such mechanisms can be facilitated by the antiinflammatory IL-10, which has been reported to be more highly expressed in the absence of TTP (34). The beneficial effects of the locally enhanced neutrophilic response, together with no overt changes in indicators of liver and kidney damage, suggest that the containment and destruction of S.pyogenes at the site of infection is the decisive step in preventing systemic disease. The importance of a neutrophilic response in defense against $S$. pyogenes underlines the significance of numerous strategies of this pathogen to evade neutrophil-mediated immunity (28).

This study establishes that the duration of the neutrophil response is restricted by TTP-promoted apoptosis of pathogenengaged neutrophils. We found that the impact of TTP on neutrophils is confined to nonhomeostatic conditions, as only infiltrating neutrophils are affected, whereas bone marrow and blood neu- trophils remain unaffected under both steady-state and infection conditions. Myeloid-specific deletion of Ttp in mice augments neutrophil activities against bacteria and prevents bacterial dissemination. The increased neutrophil response is manageable and remains without adverse health effects. These findings indicate that a temporal inhibition of TTP by pharmacological approaches might be an innovative and efficient strategy for the treatment of invasive bacterial infections.

\section{Methods}

Mice. Ttp ${ }^{f l f l}$ LysM-Cre (12), Mcl1 TG (45), and Bcl2 TG animals (46) were on a C57BL/6 genetic background. Tt $p^{f / f l}$ LysM-Cre were kept heterozygous for the Cre recombinase allele to obtain control Tt $p^{f / f l}$ $\left(\mathrm{TTP}^{\mathrm{fl} / \mathrm{fl}}\right)$ or conditional myeloid-knockout $\left(\mathrm{TTP}^{\Delta \mathrm{M}}\right)$ littermates. Mice were housed in an animal facility under specific pathogen-free conditions, and experiments were carried out using 6- to 9-week-old age- and sex-matched littermates. A solution of $10 \mathrm{mg} / \mathrm{ml}$ ketamine and $1 \mathrm{mg} / \mathrm{ml}$ xylazine (aniMedica $\mathrm{GmbH}$ ) in isotonic saline (SigmaAldrich) injected i.p. was used for experiments requiring anesthetization of mice.

Isolation and culture of peritoneal neutrophils. Neutrophils were isolated and cultured as described previously (52). Briefly, peritonitis was induced by i.p. injection of $1 \mathrm{ml}$ of $0.09 \mathrm{~g} / \mathrm{ml}$ casein (Sigma-Aldrich) in PBS with $0.5 \mathrm{mM} \mathrm{MgCl}_{2}$ and $0.9 \mathrm{mM} \mathrm{CaCl}_{2}$. Another $1 \mathrm{ml}$ was injected after 16 hours to enrich freshly recruited neutrophils. After 3 hours, the peritoneum was flushed with $10 \mathrm{ml}$ PBS containing 0.02\% EDTA. Cells were washed twice with PBS, and suspensions from corresponding mice $(n \geq 3)$ were pooled, followed by the separation and purification of neutrophils using a gradient of $3 \mathrm{ml}$ Histopaque-1119 and $3 \mathrm{ml}$ Histopaque-1077 (Sigma-Aldrich). Neutrophil-enriched phase was washed twice with PBS and cultured in RPMI (Gibco, Invitrogen, Thermo Fisher Scientific) at $37^{\circ} \mathrm{C}$ with $5 \% \mathrm{CO}_{2}$. Viability was greater than $90 \%$, and more than $95 \%$ of cells were Ly $6 \mathrm{G}^{+} \mathrm{Ly} 6 \mathrm{C}^{+} \mathrm{F} 4 / 80^{-}$ and showed typical multilobed nuclei, as determined by trypan blue staining, flow cytometry, and hematoxylin staining (Sigma-Aldrich), respectively. For in vitro stimulation and mRNA stability experiments, $3 \times 10^{6}$ to $8 \times 10^{6}$ cells were seeded per petri dish and stimulated as indicated in the figure legends.

Next-generation sequencing of RNA-seq and statistical analysis. Purified neutrophils $\left(5 \times 10^{6}\right)$ were stimulated with $10 \mathrm{ng} / \mathrm{ml} \mathrm{LPS} \mathrm{for} 4$ hours in independent triplicates. Total RNA was isolated using $1 \mathrm{ml}$ TRIzol Reagent (Life Technologies, Thermo Fisher Scientific) according to the manufacturer's protocol. Contaminating DNA was removed by digestion with $20 \mathrm{U}$ recombinant DNase I (Roche) in a total volume of 50 $\mu \mathrm{l}$ for 30 minutes at $37^{\circ} \mathrm{C}$. RNA was recovered by acid phenol/chloroform/isoamylalcohol extraction, followed by ethanol precipitation. Subsequently, RNA was used for next-generation sequencing (NGS) library preparation $(\sim 1 \mu \mathrm{g} /$ sample). PolyA enrichment was performed using the NEBNext Poly(A) mRNA Magnetic Isolation Module, followed by library preparation using the NEBNext Ultra Directional RNA Library Prep Kit (both from New England Biolabs). The library was subjected to high-throughput sequencing single-end $100 \mathrm{bp}$ on an Illumina HiSeq 2500 platform at the NGS unit of the Vienna BioCenter Core Facilities (VBCF) (http://vbcf.ac.at). Raw RNA-seq reads were preprocessed (including demultiplexing, barcode, adaptor and quality trimming, and quality control) using FastQC (http://www.bioinformatics. babraham.ac.uk/projects/fastqc/). The remaining reads were mapped 
to the GRCm38/mm10 mouse genome assembly using the genomic short-read nucleotide alignment program GSNAP, as described previously (36). We obtained more than $95 \%$ uniquely mapped reads in each sample. Sample integrity was judged by $5^{\prime}$ to $3^{\prime}$ coverage plots using sequencing quality control (RNA-SeQC). Transcripts were quantified using $\mathrm{Mix}^{2} \mathrm{RNA}$-seq data analysis software (Lexogen). The raw as well as processed data are accessible via the NCBI's Gene Expression Omnibus (GEO) database (GEO GSE77801) (http://www.ncbi.nlm. nih.gov/geo/query/acc.cgi?token=mtcvmyuehvyfroj\&acc=GSE77801). Differential expression analysis was performed in independent triplicates using DESeq2 on the basis of read counts in gene models (53). To retrieve the frequencies of AUUUA pentamers of 3 '-UTR, CDS, and 5 '-UTR for Ensembl protein-coding genes (Ensembl Genes 83), the respective regions were extracted using Ensembl Biomart $(54,55)$. The frequencies of AUUUA pentamers in each sequence region ( 5 '-UTR, CDS, 3'-UTR) for each transcript were counted using a perl script. The theoretical (expected) frequency of AUUUA was calculated for each transcript from the corresponding nucleotide composition and assuming a uniform distribution of pentamer combinations. Statistical analysis and data visualization were performed using the R project ( $R$ Development Core Team 2011; https://www.r-project.org/), perl scripts, and the ggplot2 R package (http://ggplot2.org/).

Gene ontology enrichment analysis and visualization. Genes with low expression levels (fragments per kilobase of transcripts per million fragments mapped $[\mathrm{FPKM}]<10$ ) were filtered out. Gene ontology (GO) biological process enrichment analysis for genes with significantly higher expression levels ( $\log _{2}$ fold change $[\mathrm{LFC}]>0, \mathrm{FDR}<0.05$ ) was performed using the Database for Annotation, Visualization and Integrated Discovery (DAVID) functional annotation tool, version 6.7 (56) to obtain enriched GO terms at DAVID annotation level 5. To reduce complexity, closely related GO terms were grouped. The distance between $2 \mathrm{GO}$ terms was defined as the Jaccard distance, i.e., the number of genes in the intersection of GO terms divided by the number of genes in the union of GO terms subtracted from 1 . The network was visualized using Cytoscape (57). GO terms with a Jaccard distance of 0.8 or less are connected.

Culture and preparation of bacteria. The $S$. pyogenes serotype M1 strain ISS 3348 (58) was used for all in vivo infections. S. pyogenes was grown at $37^{\circ} \mathrm{C}$ with $5 \% \mathrm{CO}_{2}$, without agitation in Todd-Hewitt broth (BD Biosciences) supplemented with $0.2 \%$ yeast extract (THY media) and on trypticase soy agar (TSA) plates containing $5 \%$ sheep's blood (BioMérieux). S. aureus RN6390 (a gift of E. Charpentier, Max Planck Institute for Infection Biology, Berlin, Germany) and E. coli DH5 $\alpha$ were grown at $37^{\circ} \mathrm{C}$ with agitation in brain-heart infusion (BHI) or on BHI agar plates and in lysogeny broth (LB) or on LB agar plates, respectively. Cell growth was turbidimetrically monitored at $620 \mathrm{~nm}$ with a microplate reader until the mid-log phase was reached. For the preparation of HK bacteria, liquid cultures were grown to the mid-log phase, washed twice with sterile PBS, and incubated for 30 minutes at $70^{\circ} \mathrm{C}$ on a thermoblock for $S$. pyogenes and S. aureus. E. coli were HK by incubation for 15 minutes at $85^{\circ} \mathrm{C}$ on a thermoblock.

Infection of animals. S. pyogenes in the stationary phase (overnight culture) were inoculated in THY media and grown at $37^{\circ} \mathrm{C}$ with $5 \%$ $\mathrm{CO}_{2}$ without agitation for 3.5 to 4 hours to reach the mid-log phase. Bacteria were harvested by centrifugation at $3,800 \mathrm{~g}$ for 6 minutes at room temperature (RT), washed twice with sterile PBS, and resuspended in isotonic saline (Sigma-Aldrich) at a concentration of $3 \times 10^{8}$ to $5 \times 10^{8} \mathrm{CFU}$ per $50 \mu \mathrm{l}$, determined by plating 10 -fold serial dilutions onto TSA plates. Animals were anesthetized, the fur of the flanks was partially shaved, and $50 \mu \mathrm{l}$ bacterial suspension was injected s.c. into the lower leg. Infected animals were monitored for their health status every 4 to 8 hours and were euthanized when they reached behavioral and humane pathophysiological endpoints. Survival was monitored for 5 days. For experiments other than those involving the survival of infected animals, the animals were euthanized 24 or 48 hours after infection, as indicated in Figure 1. To assess organ damage, the levels of blood urea, creatinine, AST, ALT, and GLDH were determined in serum samples from blood collected by heart puncture of anesthetized mice 48 hours p.i. The tests were performed by InVitro GmbH.

Histology and IHC. The infected tissue was removed at 48 hours postinfection, cut longitudinally through the lesion, and fixed overnight in $4 \%$ paraformaldehyde. The tissue was then dehydrated and embedded in paraffin, and $4-\mu \mathrm{m}$ sections were prepared using a microtome. H\&E staining was carried out following standard protocols. For Gr-1 staining, slides were deparaffinized and rehydrated, and peroxidase was blocked by incubation for 10 minutes in $3 \% \mathrm{H}_{2} \mathrm{O}_{2}$, followed by boiling for 30 minutes at $95^{\circ} \mathrm{C}$ in citric acid and sodium citrate antigen-unmasking solution. After 30 minutes of cooling down, slides were blocked with 3\% goat serum in TBS Tween-20 (TBST). Primary rat anti-mouse Gr-1 (RB6-8C5; BD Pharmingen) was applied (1:50 in TBST), and slides were incubated overnight at $4^{\circ} \mathrm{C}$. HRPconjugated secondary antibody HRP rat/mouse polymer (Envision; Dako) was added and incubated at RT for 30 minutes. After washing, a few drops of 3-amino-9-ethyl carbazole (AEC) developing reagent plus High Sensitivity Substrate (Dako) were added. To reduce unspecific staining, the sections were incubated until the appearance of a reddish color and subsequently washed. To stain $S$. pyogenes, antigens were unmasked for 10 minutes at $37^{\circ} \mathrm{C}$ with $1 \mathrm{mg} / \mathrm{ml}$ Pronase (Roche) after blocking of endogenous peroxidase activity with $\mathrm{H}_{2} \mathrm{O}_{2}$. After blocking with $3 \%$ goat serum, the sample was incubated with a rabbit antiS. pyogenes antibody (a gift from I. Julkunen, National Institute for Health and Welfare, Helsinki, Finland) (58) (1:1,000 in TBST) at RT for 1 hour. Slides were washed 3 times with TBST, followed by addition of HRP anti-rabbit polymer (Envision; Dako) as an HRPconjugated secondary antibody for 30 minutes, followed by washing. As a substrate for HRP, AEC plus High Sensitivity Substrate (Dako) were added and washed as soon as a reddish precipitate appeared. For staining other than $\mathrm{H} \& \mathrm{E}$, counterstaining was performed with incubation for a few seconds in hematoxylin solution (1:20 in doubledistilled $\mathrm{H}_{2} \mathrm{O}$ [ddH $\mathrm{de}_{2} \mathrm{O}$; Sigma-Aldrich), followed by 10 minutes of washing under running tap water. Microscopic analysis and imaging were performed using an Axio Stereomicroscope (Zeiss) and the supporting AxioVision software (Zeiss).

Determination of bacterial dissemination and lesion size. At 48 hours postinfection, the animals were deeply anesthetized, and blood was withdrawn by puncturing the heart with a 25-gauge needle, followed by 1:2 dilution of blood in sterile $\mathrm{dd}_{2} \mathrm{O}$ to lyse blood cells. The animals were then euthanized. Livers and lesions were taken and put into ice-cold PBS. After homogenization of organs and lesions, 10-fold dilution series were prepared from blood and organ samples and plated onto TSA plates. The bacterial load was determined the following day by CFU counting and normalized to volume or weight for blood, liver, and lesion, respectively. Lesion size was determined by removing the infected skin from the lower legs and measuring the diameter of the lesion, followed by calculation of the area of inflammation. 
Preparation of single-cell suspensions. Lung: After perfusing the animals with $15 \mathrm{ml}$ PBS through the right ventricle of the heart, lungs were excised, cut into small pieces, and incubated for 1 hour at $37^{\circ} \mathrm{C}$ with agitation in RPMI plus $10 \%$ FCS, penicillin/streptomycin (P/S), 1 $\mathrm{mg} / \mathrm{ml}$ collagenase 1 (Worthington), and $0.25 \mathrm{mg} / \mathrm{ml}$ DNase I (Roche). Single cells were obtained by straining the digested lungs through a 70-micron cell strainer. Spleen: Single-cell suspensions were prepared by plunging the cut spleens through a 70-micron cell strainer. Bone marrow: Cells from the femur and tibia of the hind legs of animals were flushed using DMEM, washed, and singularized by pipetting. Peritoneal exudate cells: Skin was removed, leaving the peritoneum intact. PBS (7-8 ml) was injected into euthanized animals, followed by vigorous shaking and subsequent aspiration of the cell suspension. Blood: Blood was obtained by puncturing the vena facialis. Red blood cells from blood, bone marrow, splenocytes, lung cells, and peritoneal exudate were lysed using hypotonic shock and then washed twice with PBS.

Flow cytometry. To exclude dead cells, samples were stained with Fixable Viability Dye (FVD) eFluor 506 or eFluor 780 (eBioscience), as indicated in the figure legends, prior to Fc blocking with anti-CD16/ CD32 antibody (2.4G2; BD). For pregating of hematopoietic stem cells, cells were stained with the Biotin Mouse Panel (BD Pharmingen), followed by incubation with BV786 Streptavidin (BD Biosciences). Suspensions were stained for cell-surface proteins with appropriate combinations of the following monoclonal antibodies conjugated to allophycocyanin, allophycocyanin-eFluor 780 conjugate, brilliant violet 421, brilliant violet 711, fluorescein isothiocyanate, Pacific blue, peridinin chlorophyll protein-cyanine 5.5, phycoerythrin (PE), and PE-cyanine7: anti-Ly6G (1A8, BD Biosciences), anti-Ly6C (HK1.4, ThermoFisher Scientific), anti-F4/80 (BM8, ThermoFisher Scientific), anti-CD11c (HL3, BD Biosciences), anti-MHCII (M5/114.15.2, BD Biosciences), anti-CD11b (M1/70, ThermoFisher Scientific), anti-CD45 (30-F11, ThermoFisher Scientific), anti-Gr-1 (RB6-8C5, BD Biosciences), anti-Sca1 (D7, BioLegend), anti-c-kit (2B8, BD Biosciences), anti-CD16/32 (93, ThermoFisher Scientific), anti-CD34 (RAM34, BD Biosciences), anti-SiglecF (E50-2440, BD Biosciences) and anti-FceRI $\alpha$ (MAR-1, ThermoFisher Scientific). Siglec-F and FceRI $\alpha$ were used to discriminate against eosinophils and basophils in the blood, respectively. An FITC, PE, or allophycocyanin (APC) Annexin V Apoptosis Detection Kit (eBioscience) was used according to the manufacturer's protocol. MitoStatus Red staining (BD Bioscience) was performed for 15 minutes at $37^{\circ} \mathrm{C}$ at a concentration of 20 nM. MitoSOX Red (Thermo Fisher Scientific) and a CaspGLOW Fluorescein Active Caspase-3 Staining Kit (eBioscience) were used according to the manufacturers' protocols. Dead cells doublets were excluded on the basis of fluorescence channel 2 height/area (FL2-H/ FL2-A) signal ratio or forward scatter height/area (FSC-H/FSC-A) and side scatter height/area (SSC-A/SSC-H) signal ratio. All data acquisition was performed using FACSCalibur or LSR Fortessa II cytometers interfaced with CellQuest Pro and FACSDiva (all from BD), respectively. FlowJo X software (Tree Star) was used for data analysis and graphical representation.

HK bacteria-induced peritonitis. HK S. pyogenes or S. aureus $\left(2 \times 10^{8}\right.$ $\mathrm{CFU})$ or E. coli $\left(2 \times 10^{6} \mathrm{CFU}\right)$ were injected i.p. into animals, and peritoneum was flushed after 16 or 48 hours with $10 \mathrm{ml}$ PBS containing $0.02 \%$ EDTA. The cell suspension was washed twice with PBS, and peritoneal cells were analyzed by flow cytometry or used for in vitro stimulation. For in vitro stimulation, elicited cells were first seeded onto tissue culture dishes, and after 15 minutes of incubation, all nonadherent cells were washed away.

Short-term adoptive transfer of peritoneal neutrophils. Peritoneal neutrophils from $\mathrm{TTP}^{\mathrm{f} / \mathrm{fl}}$ and $\mathrm{TTP}^{\Delta \mathrm{M}}$ mice were isolated as described above and stained according to the manufacturer's protocol with Cell Proliferation Dye eFluor 450 and eFluor 660 (eBioscience), respectively. Stained cells were counted, mixed at a 1:1 ratio, and, in total, $8 \times$ $10^{6}$ cells were injected i.p. into mice in which peritonitis was induced using $2 \times 10^{8} \mathrm{CFU}$ HK S. pyogenes 3 hours prior to transfer. Peritoneal exudate cells were harvested and analyzed 18 hours after transfer.

Phagocytosis of apoptotic neutrophils. One day before the assay, 1 $\times 10^{6}$ BMDMs were seeded onto 6-well nontissue culture plates. Peritoneal neutrophils were isolated the next day as described above, and apoptosis was induced by incubation of $1 \times 10^{6}$ neutrophils $/ \mathrm{ml}$ with 0.1 $\mathrm{mM} \mathrm{H}_{2} \mathrm{O}_{2}$ (Sigma-Aldrich) and $30 \mathrm{ng} / \mathrm{ml}$ PMA for 1 hour as described previously (40). Apoptotic and nonapoptotic neutrophils were stained with $20 \mathrm{ng} / \mathrm{ml}$ pHrodo Red, SE (Thermo Fisher Scientific) according to the manufacturer's protocol at a concentration of $10 \times 10^{6}$ cells $/ \mathrm{ml}$. Stained neutrophils were added to BMDMs at a 1:2 ratio, and plates were synchronized by spinning for 1 minute at $300 \mathrm{~g}$. As a negative control for phagocytosis, cytochalasin D from Zygosporium mansonii (Sigma-Aldrich) was added 1 hour prior to the addition of apoptotic neutrophils at a concentration of $1 \mu \mathrm{g} / \mathrm{ml}$. After a 1.5-hour incubation at $37^{\circ} \mathrm{C}$, cells were scraped and analyzed by flow cytometry.

Migration assay. Purified peritoneal neutrophils $\left(3 \times 10^{5}\right)$ were thoroughly and gently mixed with freshly prepared and buffered collagen solution (Nutragen from Advanced BioMatrix, bovine type I collagen), with a final collagen concentration of $3.2 \mathrm{mg} / \mathrm{ml}$. Cell suspension in the collagen solution was incubated in custom-built migration chambers (2-mm-thick) at $37^{\circ} \mathrm{C}$. After polymerization, the gels were overlaid with cell culture medium, with or without recombinant CXCL1 (100 ng/ml; PeproTech), and subsequently imaged with bright-field video microscopes equipped with custom-built climate chambers $(5 \%$ $\mathrm{CO}_{2}, 37^{\circ} \mathrm{C}$, humidified) for 6 hours at 30 seconds per frame. Images were then processed with ImageJ, version $1.51 \mathrm{~h}(\mathrm{NIH})$, single cells were tracked with the ImageJ TrackMate plugin, and tracks with low detection quality were excluded from the analysis. Tracking data were processed for plotting and statistics in R. Pairwise unpaired, 2-tailed Student's $t$ tests and ANOVA were performed for statistical analysis.

$R N A$ isolation, reverse transcription, and quantitative PCR. For RNA isolation, cells were lysed using Isol-RNA Lysis Reagent (5 Prime), and RNA was isolated following standard protocols. Contaminating DNA was digested with 10 U DNase I (Roche), and cDNA was synthesized using Mu-MLV Reverse Transcriptase (Fermentas) by PolyA priming with oligo $(\mathrm{dT})_{18}$ primers (Eurofins Genomics). Quantitative PCRs (qPCRs) were run on a Realplex Mastercycler (Eppendorf) and monitored by the SYBR Green method using HOT FIREPol EvaGreen qPCR Supermix (Medibena). mRNA levels were calculated using a 2-fold standard dilution series derived from the samples. Mcl1 was detected using QuantiTect Primer Assays (QIAGEN; catalog QT01764644). The primer sequences for RplpO (used for normalization), Cxcl2, Hprt, Il1b, Il6, $T n f$, and Ttp (also known as Zfp36) are listed in Supplemental Table 3.

Determination of $m R N A$ stability. mRNA decay assays were carried out as described previously (12). In brief, cells were seeded and washed after the majority of cells were adherent. Cells were stimulated with LPS, as indicated in the figure legends. Medium was then removed, and fresh medium containing the transcription inhibitor ActD and p38 
MAPK inhibitor SB203580 (to obtain highly active TTP) (12) was added for the durations indicated in Figure 6C, followed by RNA isolation. mRNA levels were analyzed by reverse transcription quantitative PCR (RT-qPCR). The half-lives of transcripts were calculated with a fitted exponential curve.

Differentiation and culture of BMDMs. BMDMs were grown in L cellderived CSF-1 as described previously (13). Mice used for bone marrow collection were 8-12 weeks old.

UV crosslinking and RNA-IP. TTP-RNA interactions were analyzed using UV crosslinking, followed by IP using specific antibodies. BMDMs $\left(2 \times 10^{7}\right.$ cells $)$ were cultured in medium supplemented with $100 \mu \mathrm{M}$ 4-thiouridine (Sigma-Aldrich) for 16 hours prior to the experiment. Cells were stimulated with LPS $(10 \mathrm{ng} / \mathrm{ml})$ for 6 hours, followed by washing with PBS and crosslinking with $365 \mathrm{~nm}$ UV light at $0.15 \mathrm{~J} / \mathrm{cm}^{2}$. Subsequently, cells were harvested in lysis buffer $(50 \mathrm{mM}$ Tris-HCl, pH 7.4, 100 mM NaCl, 1\% NP40, 0.1\% SDS, 0.5\% sodium deoxycholate, and protease inhibitor cocktail) (Roche). Cell lysate was treated with 10 U DNase I (Roche). TTP was immunoprecipitated using TTP rabbit antiserum (12) bound to Protein G Dynabeads (Invitrogen, Thermo Fisher Scientific) for 3 hours at $4^{\circ} \mathrm{C}$. Beads were washed with lysis buffer and subsequently with washing buffer (20 mM Tris$\mathrm{HCl}, \mathrm{pH}$ 7.4, $10 \mathrm{mM} \mathrm{MgCl}_{2}$, and 0.2\% Tween-20). RNA was eluted from RNA-protein complexes by Proteinase K (Roche) digestion. RNA was purified using phenol/chloroform/isoamyl alcohol (25:24:1, v/v, $\mathrm{pH}$ 4.5) extraction, followed by ethanol precipitation. Immunoprecipitated RNA was analyzed by RT-qPCR and normalized to input.

Western blot analysis. After treatment, $5 \times 10^{6}$ to $8 \times 10^{6}$ neutrophils were lysed using $100 \mu \mathrm{l}$ NuPAGE LDS sample buffer (Invitrogen, Thermo Fisher Scientific), followed by sonication 3 times for $30 \mathrm{sec}-$ onds and heating at $95^{\circ} \mathrm{C}$ for 15 minutes prior to loading. Western blotting was performed as previously described (12). For signal detection, the Odyssey Infrared Imaging System and Image Studio (LI-COR Biosciences) were used. GelAnalyzer 2010a was used for quantification.

Reagents. Rabbit antibody against TTP was used as described previously (12). Tubulin (DM1A; Sigma-Aldrich), ERK (16/ERK; BD), and MCL-1 (D35A5; Cell Signaling Technology) antibodies were used as recommended. LPS (10 ng/ml) from E. coli 055:B5 (Sigma-Aldrich) or $5 \mu \mathrm{g} / \mathrm{ml}$ lipoteichoic acid (LTA) from $S$. pyogenes (Sigma-Aldrich) was used for stimulation of cells, and ActD and SB203580 (both from SigmaAldrich) were used at a concentration of $5 \mathrm{mg} / \mathrm{ml}$ and $4 \mu \mathrm{M}$, respectively.
Statistics. Exploratory data analysis, visualization, and statistical testing were performed using GraphPad Prism 5 (GraphPad Software) or R Project (R Development Core Team 2011) with the Mantel-Cox log-rank test, unpaired 2-tailed Student's $t$ test, MannWhitney $U$ test, linear regression model, $\chi^{2}$ test, ANOVA, or Pearson's and Spearman's correlation tests, as indicated in the figure legends. For parametric or nonparametric statistical testing, the mean or median is depicted by error bars, respectively. A $P$ value of less than 0.05 was considered significant.

Study approval. All animal experiments were discussed with the institutional ethics committee and performed in accordance with Austrian law (BGBl. I Nr. 114/2012) and with the guidelines of the German Society of Laboratory Animals (GV-SOLAS). All animal experimental protocols were approved and authorized by the Austrian Ministry of Science (BMWF-66.006/0006-II/3b/2013, issued to PK).

\section{Author contributions}

FE, VS, ST, MS, and PK designed experiments. FE, VS, ST, and MI conducted experiments. FE, VS, ST, MI, FK, NG, LK, MS, and PK analyzed data. AV provided reagents. FE and PK wrote the manuscript.

\section{Acknowledgments}

We are grateful to Susanne Cory (Walter and Eliza Hall Institute of Medical Research, Melbourne, Australia) for providing the transgenic Mcl1 mice. We thank Marton Janos (Max F. Perutz Laboratories, University of Vienna, Vienna, Austria), and Krzysztof Chylinski (Vienna Biocenter Core Facilities, Vienna, Austria) for critical reading of the manuscript. This work was supported by grants from the Austrian Science Fund (FWF) (P27538-B21, I1621-B22, and SFB 43, to PK); by funding from the European Union Seventh Framework Programme Marie Curie Initial Training Networks (FP7-PEOPLE-2012-ITN) for the project INBIONET (INfection BIOlogy Training NETwork under grant agreement PITN-GA-2012-316682; and by a joint research cluster initiative of the University of Vienna and the Medical University of Vienna.

Address correspondence to: Pavel Kovarik, Dr. Bohr-Gasse 9, 1030 Vienna, Austria. Phone: 43.1.4277.54608; Email: pavel. kovarik@univie.ac.at.
1. Kolaczkowska E, Kubes P. Neutrophil recruitment and function in health and inflammation. Nat Rev Immunol. 2013;13(3):159-175.

2. Kruger P, et al. Neutrophils: Between host defence, immune modulation, and tissue injury. PLoS Pathog. 2015;11(3):e1004651.

3. Koedel U, et al. Apoptosis is essential for neutrophil functional shutdown and determines tissue damage in experimental pneumococcal meningitis. PLoS Pathog. 2009;5(5):e1000461.

4. Bardoel BW, Kenny EF, Sollberger G, Zychlinsky A. The balancing act of neutrophils. Cell Host Microbe. 2014;15(5):526-536.

5. Kafasla P, Skliris A, Kontoyiannis DL. Posttranscriptional coordination of immunological responses by RNA-binding proteins.

Nat Immunol. 2014;15(6):492-502.

6. Kovarik P, Ebner F, Sedlyarov V. Posttranscrip- tional regulation of cytokine expression. Cytokine. 2017;89:21-26.

7. Carpenter S, Ricci EP, Mercier BC, Moore MJ, Fitzgerald KA. Post-transcriptional regulation of gene expression in innate immunity. Nat Rev Immunol. 2014;14(6):361-376.

8. Wells ML, Perera L, Blackshear PJ. An Ancient Family of RNA-Binding Proteins: Still Important! Trends Biochem Sci. 2017;42(4): 285-296.

9. Brook M, et al. Posttranslational regulation of tristetraprolin subcellular localization and protein stability by p38 mitogen-activated protein kinase and extracellular signal-regulated kinase pathways. Mol Cell Biol. 2006;26(6):2408-2418.

10. Hitti E, et al. Mitogen-activated protein kinase-activated protein kinase 2 regulates tumor necrosis factor mRNA stability and translation mainly by altering tristetraprolin expression, stability, and binding to adenine/uridine-rich element. Mol Cell Biol. 2006;26(6):2399-2407.

11. Stoecklin G, et al. MK2-induced tristetraprolin: 14-3-3 complexes prevent stress granule association and ARE-mRNA decay. EMBO J. 2004;23(6):1313-1324.

12. Kratochvill F, et al. Tristetraprolin-driven regulatory circuit controls quality and timing of mRNA decay in inflammation. Mol Syst Biol. 2011;7:560.

13. Schaljo B, et al. Tristetraprolin is required for full anti-inflammatory response of murine macrophages to IL-10. J Immunol. 2009;183(2):1197-1206.

14. Taylor GA, et al. A pathogenetic role for TNF alpha in the syndrome of cachexia, arthritis, and autoimmunity resulting from tristetraprolin (TTP) deficiency. Immunity. 1996;4(5):445-454. 15. Molle C, et al. Tristetraprolin regulation of 
interleukin 23 mRNA stability prevents a spontaneous inflammatory disease. J Exp Med. 2013;210(9):1675-1684.

16. Qiu LQ, Stumpo DJ, Blackshear PJ. Myeloidspecific tristetraprolin deficiency in mice results in extreme lipopolysaccharide sensitivity in an otherwise minimal phenotype. JImmunol. 2012;188(10):5150-5159.

17. Dzhagalov I, Dunkle A, He YW. The anti-apoptotic Bcl-2 family member Mcl-1 promotes $\mathrm{T}$ lymphocyte survival at multiple stages. JImmunol. 2008;181(1):521-528.

18. Steimer DA, Boyd K, Takeuchi O, Fisher JK, Zambetti GP, Opferman JT. Selective roles for antiapoptotic MCL-1 during granulocyte development and macrophage effector function. Blood. 2009;113(12):2805-2815.

19. Wessels MR. Clinical practice. Streptococcal pharyngitis. N EnglJMed. 2011;364(7):648-655.

20. Musser JM, Shelburne SA. A decade of molecular pathogenomic analysis of group A Streptococcus. JClin Invest. 2009;119(9):2455-2463.

21. Ashbaugh CD, Warren HB, Carey VJ, Wessels MR. Molecular analysis of the role of the group A streptococcal cysteine protease, hyaluronic acid capsule, and $\mathrm{M}$ protein in a murine model of human invasive soft-tissue infection. JClin Invest. 1998;102(3):550-560.

22. Medina E. Murine model of cutaneous infection with Streptococcus pyogenes. Methods Mol Biol. 2010;602:395-403.

23. Navarini AA, et al. Innate immune-induced depletion of bone marrow neutrophils aggravates systemic bacterial infections. Proc Natl Acad Sci US A. 2009;106(17):7107-7112.

24. Buchanan JT, et al. DNase expression allows the pathogen group A Streptococcus to escape killing in neutrophil extracellular traps. Curr Biol. 2006;16(4):396-400.

25. Goldmann O, Rohde M, Chhatwal GS, Medina E. Role of macrophages in host resistance to group A streptococci. Infect Immun. 2004;72(5):2956-2963.

26. Mishalian I, et al. Recruited macrophages control dissemination of group A Streptococcus from infected soft tissues. Jimmunol. 2011;187(11):6022-6031.

27. Castiglia V, et al. Type I Interferon Signaling Prevents IL-1 $\beta$-Driven Lethal Systemic Hyperinflammation during Invasive Bacterial Infection of Soft Tissue. Cell Host Microbe. 2016;19(3):375-387.

28. Döhrmann S, Cole JN, Nizet V. Conquering Neutrophils. PLoS Pathog. 2016;12(7):e1005682.

29. Davies LC, Jenkins SJ, Allen JE, Taylor PR. Tissue-resident macrophages. Nat Immunol.
2013;14(10):986-995.

30. Shi C, Pamer EG. Monocyte recruitment during infection and inflammation. Nat Rev Immunol. 2011;11(11):762-774.

31. Summers C, Rankin SM, Condliffe AM, Singh N, Peters AM, Chilvers ER. Neutrophil kinetics in health and disease. Trends Immunol. 2010;31(8):318-324.

32. Brummer E, McEwen JG, Stevens DA. Fungicidal activity of murine inflammatory polymorphonuclear neutrophils: comparison with murine peripheral blood PMN. Clin Exp Immunol. 1986;66(3):681-690.

33. Emmons J, et al. Identification of TTP mRNA targets in human dendritic cells reveals TTP as a critical regulator of dendritic cell maturation. RNA. 2008;14(5):888-902.

34. Stoecklin G, et al. Genome-wide analysis identifies interleukin-10 mRNA as target of tristetraprolin. J Biol Chem. 2008;283(17):11689-11699.

35. Tiedje C, et al. The RNA-binding protein TTP is a global post-transcriptional regulator of feedback control in inflammation. Nucleic Acids Res. 2016;44(15):7418-7440.

36. Sedlyarov V, et al. Tristetraprolin binding site atlas in the macrophage transcriptome reveals a switch for inflammation resolution. Mol Syst Biol. 2016;12(5):868.

37. Zhang X, et al. Gene expression in mature neutrophils: early responses to inflammatory stimuli. JLeukoc Biol. 2004;75(2):358-372.

38. Brookes PS, Yoon Y, Robotham JL, Anders MW, Sheu SS. Calcium, ATP, and ROS: a mitochondrial love-hate triangle. Am J Physiol, Cell Physiol. 2004;287(4):C817-C833.

39. Kroemer G, Galluzzi L, Brenner C. Mitochondrial membrane permeabilization in cell death. Physiol Rev. 2007;87(1):99-163.

40. Tsurubuchi T, Aratani Y, Maeda N, Koyama H. Retardation of early-onset PMA-induced apoptosis in mouse neutrophils deficient in myeloperoxidase. J Leukoc Biol. 2001;70(1):52-58.

41. Opferman JT, Letai A, Beard C, Sorcinelli MD, Ong CC, Korsmeyer SJ. Development and maintenance of $B$ and $T$ lymphocytes requires antiapoptotic MCL-1. Nature. 2003;426(6967):671-676.

42. Dzhagalov I, St John A, He YW. The antiapoptotic protein Mcl-1 is essential for the survival of neutrophils but not macrophages. Blood. 2007;109(4):1620-1626.

43. Moulding DA, Akgul C, Derouet M, White MR, Edwards SW. BCL-2 family expression in human neutrophils during delayed and accelerated apoptosis. JLeukoc Biol. 2001;70(5):783-792.
44. Ertel F, Nguyen M, Roulston A, Shore GC. Programming cancer cells for high expression levels of Mcl1. EMBO Rep. 2013;14(4):328-336.

45. Campbell KJ, et al. Elevated Mcl-1 perturbs lymphopoiesis, promotes transformation of hematopoietic stem/progenitor cells, and enhances drug resistance. Blood. 2010;116(17):3197-3207.

46. Ogilvy S, Metcalf D, Print CG, Bath ML, Harris AW, Adams JM. Constitutive Bcl-2 expression throughout the hematopoietic compartment affects multiple lineages and enhances progenitor cell survival. Proc Natl Acad Sci USA. 1999;96(26):14943-14948.

47. Elliott MR, et al. Nucleotides released by apoptotic cells act as a find-me signal to promote phagocytic clearance. Nature. 2009;461(7261):282-286.

48. Croker BA, et al. Fas-mediated neutrophil apoptosis is accelerated by Bid, Bak, and Bax and inhibited by Bcl-2 and Mcl-1. Proc Natl Acad Sci USA. 2011;108(32):13135-13140.

49. Hamasaki A, et al. Accelerated neutrophil apoptosis in mice lacking A1-a, a subtype of the bcl-2-related A1 gene. J Exp Med. 1998;188(11):1985-1992.

50. Kirschnek S, et al. Molecular analysis of neutrophil spontaneous apoptosis reveals a strong role for the pro-apoptotic BH3-only protein Noxa. Cell Death Differ. 2011;18(11):1805-1814.

51. Miller LS, Cho JS. Immunity against Staphylococcus aureus cutaneous infections. Nat Rev Immunol. 2011;11(8):505-518.

52. Watt SM, Burgess AW, Metcalf D. Isolation and surface labeling of murine polymorphonuclear neutrophils. J Cell Physiol. 1979;100(1):1-21.

53. Love MI, Huber W, Anders S. Moderated estimation of fold change and dispersion for RNA-seq data with DESeq2. Genome Biol. 2014;15(12):550.

54. Cunningham F, et al. Ensembl 2015. Nucleic Acids Res. 2015;43(Database issue):D662-D669.

55. Guberman JM, et al. BioMart Central Portal: an open database network for the biological community. Database (Oxford). 2011;2011:bar0 41.

56. Huang da W, Sherman BT, Lempicki RA. Systematic and integrative analysis of large gene lists using DAVID bioinformatics resources. Nat Protoc. 2009;4(1):44-57.

57. Smoot ME, Ono K, Ruscheinski J, Wang PL, Ideker T. Cytoscape 2.8: new features for data integration and network visualization. Bioinformatics. 2011;27(3):431-432.

58. Gratz N, et al. Type I interferon production induced by Streptococcus pyogenes-derived nucleic acids is required for host protection. PLoS Pathog. 2011;7(5):e1001345. 